\title{
Two-frequency analysis of interactions elicited by Vernier stimuli
}

\author{
JONATHAN D. VICTOR AND MARY M. CONTE \\ Department of Neurology and Neuroscience, Weill Medical College of Cornell University, New York \\ (Received May 26, 2000; Accepted August 3, 2000)
}

\begin{abstract}
In five subjects, we measured visual evoked potentials (VEPs) elicited by Vernier targets in which the contrast of the two components of the stimuli were modulated by sinusoids at distinct frequencies $f_{1}$ and $f_{2}$. This approach allows for the extraction of VEP signatures of spatial interactions, namely, responses at intermodulation frequencies $n_{1} f_{1}+n_{2} f_{2}$, without the need to introduce motion into the stimulus. The most prominent interactions were at the sum frequency $f_{1}+f_{2}$, and, for frequency pairs that were sufficiently separated, the difference frequency $f_{1}-f_{2}$. These responses had a systematic dependence on the temporal parameters of the stimulus, corresponding to an effective latency of 145 to $165 \mathrm{~ms}$. Fourth-order interactions were also detected, particularly at the frequencies $2 f_{1} \pm 2 f_{2}$. These VEP signatures of interaction were similar to interactions seen for colinear line segments separated by a gap. Thus, for Vernier stimuli devoid of motion, VEP signatures of interaction are readily detected but are not specific to hyperacuity displacements. The distribution of interactions across harmonic orders is consistent with local rectification preceding the spatial interactions. Their effective latencies and dependence on spatial parameters are consistent with interactions within V1 receptive fields or mediated by horizontal connections between cells with a similar orientation tuning within V1.
\end{abstract}

Keywords: VEP, Hyperacuity, Nonlinear analysis, Two-sinusoid method

\section{Introduction}

In Vernier alignment paradigms, observers are able to make exceptionally fine positional judgments (Westheimer, 1981; Klein \& Levi, 1985). These judgments are in the hyperacuity range (at a finer grain than the photoreceptor lattice). The information required for these judgments must be present in the retinal output. Indeed, the contrast sensitivity of individual retinal ganglion cells would support a behavioral Vernier threshold much lower than what is observed (Shapley \& Victor, 1986), if the contrast changes signalled by a single retinal ganglion cell were efficiently interpreted as positional shifts. However, changes in firing rates of a single ganglion cell, though precise, do not necessarily indicate positional shifts. Firing rate changes can only be interpreted as shifts in the context of appropriate activity in nearby neurons. Inefficiency in this cortical analysis, rather than the limits of spatial pooling per se, controls Vernier thresholds.

Current understanding of the computations underlying shortrange hyperacuity is based on general-purpose idealized cortical neurons (i.e., quasilinear neurons with oriented, Gabor-like receptive fields). These computational models (Klein \& Levi, 1985; Wilson, 1986) as well as more recent elaborations of them (Carney

Address correspondence and reprint requests to: Jonathan D. Victor, Department of Neurology and Neuroscience, Weill Medical College of Cornell University, 1300 York Avenue, New York, NY 10021, USA. Email: jdvicto@med.cornell.edu
\& Klein, 1999), suffice to account for the basic features of hyperacuity performance, although recent masking studies suggest the existence of special-purpose mechanisms specifically tuned to local Vernier targets (Levi et al., 2000).

Visual evoked potentials (VEPs) represent a bridge between human psychophysics and neural activity (Regan, 1989). VEP correlates of Vernier acuity have been identified in two kinds of paradigms: making and breaking of line segments (Levi et al., 1983; Steinman et al., 1985; Zak \& Berkley, 1986) and spatial shifts of gratings (Norcia et al., 1999). Controls in these studies indicate that the VEPs are specific to small relative motions, not motion per se. Nevertheless, because of the nature of the stimuli, the relationship of the neural mechanisms that generate these VEPs to those that are involved in Vernier tasks without motion is unclear.

Most psychophysical studies of Vernier acuity involve stationary stimuli, but VEPs can only be elicited by stimuli that change in time. To make a more direct connection between VEPs and such studies, it is necessary to use stimuli that are dynamic but stationary. We therefore chose to apply the "two-sinusoid" method (Zemon \& Ratliff, 1984; Regan \& Regan, 1988a,b) to stationary Vernier targets. To do this, we modulated the contrast of each bar of a two-bar Vernier target by a different sinusoidal signal. The resulting VEP contained Fourier components not just at these modulation frequencies and their harmonics, but also at various intermodulation frequencies. The latter components could only be generated by neural mechanisms that received inputs from both bars, and only if these inputs interacted. By examining how the 
VEP depended on the Vernier offset between the two bars and on colinear displacements across a gap, the specificity of the interaction for Vernier offsets could be assayed. By examining the characteristics of the Fourier components elicited by these stimuli, dynamical models (Victor \& Conte, 1999a) for the interactions underlying Vernier acuity could be tested.

\section{Methods}

\section{Visual stimuli}

Fig. 1A diagrams a typical stimulus. It is composed of a regular array of Vernier targets, each consisting of two bars. Across their width, the bars had a Gaussian profile, so that subpixel vertical displacements $d$ could be produced (Krauskopf \& Farell, 1991; Victor \& Conte, 1999a). We used six such vertical displacements $d(0,0.5,1,2,4$, or $8 \mathrm{~min})$, as diagrammed in Fig. 1B. Bars had a length of $60 \mathrm{~min}$ and a width (full width at half-maximum) of $7.5 \mathrm{~min}$ (as in Fig. 1A) or $1.7 \mathrm{~min}$. For the wide bars, the stimulus consisted of a $14 \times 5$ array of Vernier targets; for the narrow bars, an $18 \times 5$ array was used. The minimum distance between bars of separate Vernier targets was $21.5 \mathrm{~min}$ (vertical) and $30 \mathrm{~min}$ (horizontal). At the largest displacement, the vertical distance between bars of separate targets was $13.5 \mathrm{~min}$.

In one experiment, a horizontal gap $g$ was introduced between the bars. The ends of the bars were sharp, so that the gaps $g$ were limited to integer multiples of the pixel size $(0,1,2,4$, or $8 \mathrm{~min})$. At the largest gap size, the horizontal distance between bars of separate targets was $22 \mathrm{~min}$. To prevent interactions between bars of neighboring targets from dominating the interactions within a target, substantially larger displacements or gaps were not used.

Within each target, each bar was assigned to one of two frequencies $f_{1}$ and $f_{2}$ and contrast-modulated with a maximum con- trast of 1.0. This assignment was made as shown in Fig. 1. For example, if the left bar was assigned to $f_{1}$ in one target, then the right bar was assigned to $f_{1}$ in the neighboring targets. This scheme reduced the sense of global apparent motion that would be generated if the assignment was identical in all targets. The frequencies $f_{1}$ and $f_{2}$ were chosen to be relatively prime integer multiples (see Fig. 2) of a common fundamental frequency $F_{0}=1 / 10.24 \mathrm{~Hz}$. For example, the frequency pair with $N_{1}=31$ and $N_{2}=33$ corresponds to $f_{1}=N_{1} F_{0}=3.027 \mathrm{~Hz}$ and $f_{2}=N_{2} F_{0}=3.223 \mathrm{~Hz}$.

\section{Display}

Stimuli were produced on a Sony Multiscan 17 seII monitor, with signals driven by a PC-controlled Cambridge Research VSG2/3 graphics processor. The resulting $768 \times 1024$ pixel display had a mean luminance of $47.2 \mathrm{~cd} / \mathrm{m}^{2}$, a refresh rate of $100 \mathrm{~Hz}$, and subtended $11.2 \times 13.3 \mathrm{deg}(1 \mathrm{~min} / \mathrm{pixel})$ at the viewing distance of $114 \mathrm{~cm}$. The intensity versus voltage behavior of the monitor was linearized by photometry and lookup table adjustments as provided by the VSG software.

\section{Subjects and VEP recording}

Studies were conducted in five normal subjects (two male, three female) who ranged in age from 28 to 46 years, and had visual acuities (with correction if necessary) of $20 / 20$ or better. All were experienced psychophysical and VEP subjects. The three nonauthor subjects were naive to the purpose of the experiments. Viewing was binocular, and subjects were instructed to fixate a dot positioned in the center of the display.

Scalp signals were obtained from standard gold-cup electrodes, applied to the scalp with Nihon-Kohden electrolyte paste at $C_{z}(+)$ and $O_{z}(-)$. Electroencephalographic activity was amplified 10,000-

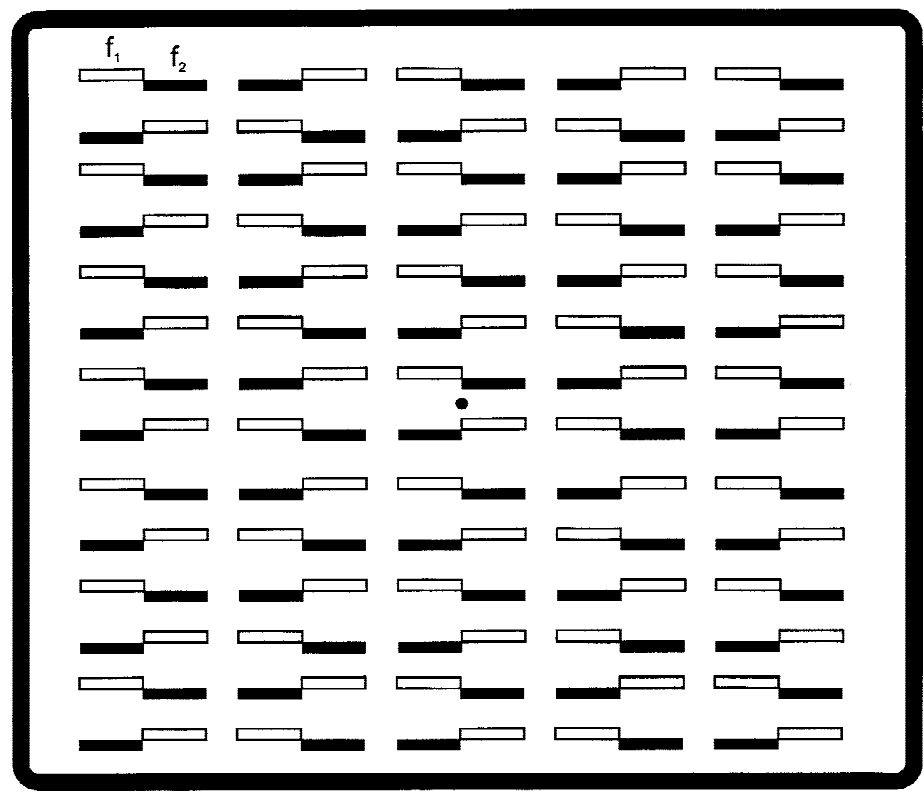

A.

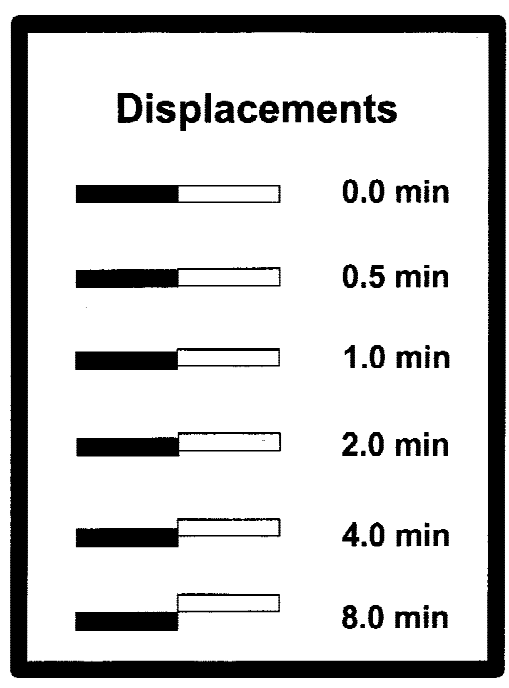

B.

Fig. 1. (A) An example of the typical stimulus, consisting of a $14 \times 5$ array of Vernier bars $(7.5 \mathrm{~min} \times 60 \mathrm{~min})$, with an offset $d$ of $8 \mathrm{~min}$ and a gap $g$ of $0 \mathrm{~min}$. Bars were sinusoidally contrast-modulated at one of two frequencies, with bars diagrammed as white modulated at $f_{1}$ and bars diagrammed as black modulated at $f_{2}$. (B) Diagram of the six Vernier displacements used. 


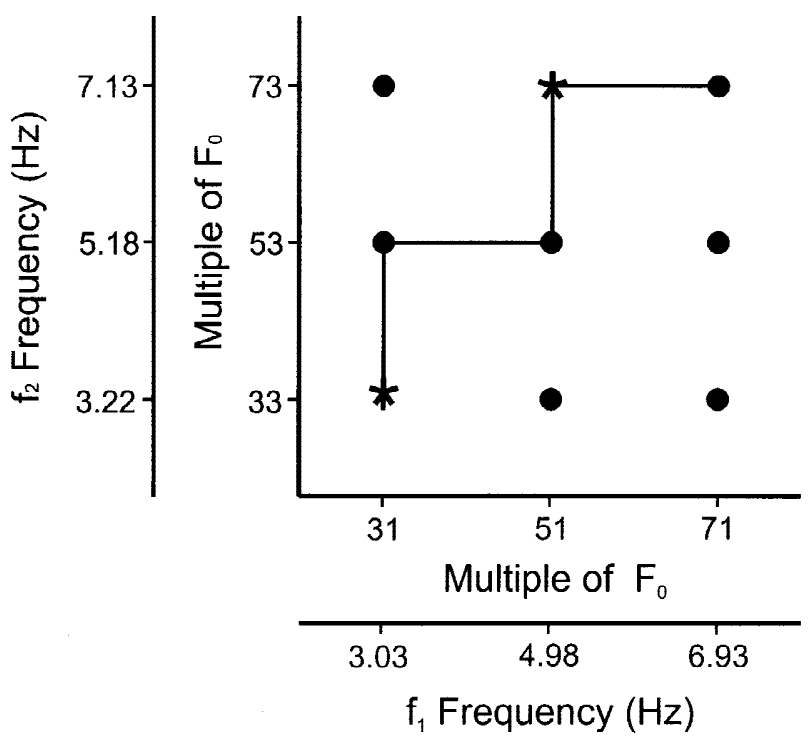

Fig. 2. The frequency pairs $\left(f_{1}, f_{2}\right)$ used to modulate the components of the Vernier target. For each frequency pair, the frequencies are large relatively prime integer multiples of a common fundamental frequency $F_{0}=1 / 10.24 \mathrm{~Hz}$.

fold, filtered ( 0.1 to $100 \mathrm{~Hz}$ ) and digitized at $400 \mathrm{~Hz}$, four times the frame rate, by hardware that was synchronized to the VSG stimulus control.

Experimental sessions were organized into eight blocks of 36-s trials, each of which contained one example of each condition, in randomized order. The parameters varied within the block included Vernier displacement $d$, gap $g$, bar width, and the $\left(f_{1}, f_{2}\right)$ frequency pair (Fig. 2). In one experiment in which a single bar width and all nine frequency pairs were used, Vernier displacement $d$ and gap $g$ were held constant at 0 . In experiments in which Vernier displacement and/or gap were parametrically varied, a restricted set of frequency pairs (“*” or connected by a line in Fig. 2) were used, as detailed below.

Raw voltage signals from each trial were inspected on line, and trials that contained evidence of artifact were discarded and repeated. (This was typically less than $15 \%$ of the trials). The initial $5 \mathrm{~s}$ of each trial was discarded to avoid transient effects related to stimulus onset. The remaining $30.72 \mathrm{~s}$ of each trial was subdivided into three nonoverlapping epochs, each of which contained a single common period $T=1 / F_{0}=10.24 \mathrm{~s}$ of the visual stimulus (with each sinusoid peaking at the beginning of the epoch), and 4096 voltage samples. Fourier components of the VEP were calculated at a range of combinations frequencies $n_{1} f_{1}+n_{2} f_{2}\left(n_{1}, n_{2}\right.$ integers) from each of the $M=24$ epochs (eight trials, three segments per trial). We denote the estimate of the response component at $n_{1} f_{1}+$ $n_{2} f_{2}$ derived from the epoch $m$ by $A_{m}\left(n_{1}, n_{2}\right)$. That is, $A_{m}\left(n_{1}, n_{2}\right)$ is a complex Fourier component, given by

$$
A_{m}\left(n_{1}, n_{2}\right)=\frac{2}{T} \int_{0}^{T} V_{m}(t) \exp \left[-2 \pi \iota\left(n_{1} f_{1}+n_{2} f_{2}\right) t\right\} \mathrm{d} t,
$$

where $V_{m}(t)$ is the linearly detrended electroencephalogram (EEG) voltage (i.e. the raw EEG voltage with the best-fitting straight line subtracted, to eliminate slow drifts) sampled at time $t$ into the $m$ th epoch. Note that $A_{m}\left(n_{1}, n_{2}\right)$ and $A_{m}\left(-n_{1},-n_{2}\right)$ are complex con- jugates, so it suffices to consider the combination frequencies $\left(n_{1}, n_{2}\right)$ for which $n_{1}>0$ and $n_{2}$ is positive, negative, or zero, or for which $n_{1}=0$, and $n_{2}>0 . A_{m}(1,0)$ and $A_{m}(0,1)$ are, respectively, the Fourier components at the two input frequencies $f_{1}$ and $f_{2}$. The response amplitude is given by the magnitude of the complex number $A_{m}\left(n_{1}, n_{2}\right)$, and the response phase is given by the phase of $A_{m}\left(n_{1}, n_{2}\right)$. The phases of the input sinusoids were arranged so that they always peaked at the beginning of each epoch. Consequently, for a linear system, the response phases as determined by eqn. (1) correspond to the phases of the transfer function at the frequencies $f_{1}$ and $f_{2}$.

For each combination frequency $\left(n_{1}, n_{2}\right)$, the estimates $A_{m}\left(n_{1}, n_{2}\right)$ were pooled across the $M(=24)$ epochs by vector averaging to provide an overall response estimate $A\left(n_{1}, n_{2}\right)$ :

$$
A\left(n_{1}, n_{2}\right)=\frac{1}{M} \sum_{m=1}^{M} A_{m}\left(n_{1}, n_{2}\right)
$$

The duration of each epoch was long enough ( $>8 \mathrm{~s}$, Victor \& Mast, 1991) for the quantities $A_{m}\left(n_{1}, n_{2}\right)$ to be regarded as statistically independent response estimates. Their variability is quantified by

$$
P\left(n_{1}, n_{2}\right)=\frac{1}{M-1} \sum_{m=1}^{M}\left|A_{m}\left(n_{1}, n_{2}\right)-A\left(n_{1}, n_{2}\right)\right|^{2},
$$

the mean-squared scatter of the individual estimates $A_{m}\left(n_{1}, n_{2}\right)$ about their mean. (The denominator $M-1$ rather than $M$ corrects for the loss of a degree of freedom due to the estimation of the mean response $A\left(n_{1}, n_{2}\right)$ from the individual estimates $\left.A_{m}\left(n_{1}, n_{2}\right)\right)$. The ratio $\left|A\left(n_{1}, n_{2}\right)\right|^{2} / P\left(n_{1}, n_{2}\right)$ is the $T_{\text {circ }}^{2}$ statistic (Victor \& Mast, 1991) and thus provides a way to determine whether a significant response is present. Under the null hypothesis that the quantities $A_{m}\left(n_{1}, n_{2}\right)$ scatter randomly around zero, this ratio is distributed according to $F_{[2,2 M-2]}$. Moreover, whether or not a significant response is present, the assumption that the observed epoch-to-epoch fluctuations in the response estimates $A_{m}\left(n_{1}, n_{2}\right)$ are due to additive combination of a fixed VEP response $A\left(n_{1}, n_{2}\right)$ and independent ongoing EEG activity implies that the variabilities $P\left(n_{1}, n_{2}\right)$ will be proportional to the power of the EEG background at the frequency $n_{1} f_{1}+n_{2} f_{2}$ (Mast \& Victor, 1991).

The above calculations were performed for combination frequencies up to order 8 (i.e. for non-negative integers $n_{1}$ and $n_{2}$ for which $n_{1}+n_{2} \leq 8$ ), but our analysis will be restricted to combination frequencies up to order 6 , since Fourier components at higher orders were not significant by the $T_{\text {circ }}^{2}$ statistic. Up to order 6 , there are 42 distinct response frequencies, 12 of which are harmonics of each of the two input frequencies, and 30 of which are interaction frequencies.

The general qualitative interpretation of the Fourier components $A\left(n_{1}, n_{2}\right)$ is discussed in detail by Regan and Regan (1988b) and Regan (1990), and is briefly summarized here. Linear systems are expected to produce significant response components only at the first-order frequencies $\left(n_{1}, n_{2}\right)=(1,0)$ and $(0,1)$. Nonlinear systems would be expected to produce significant response components $A\left(n_{1}, n_{2}\right)$ for values of $n_{1}$ and/or $n_{2}$ greater than 1 . If the nonlinearities affect signals from each stimulus component separately, significant responses would be observed for $\left(n_{1}, n_{2}\right)=$ $(2,0),(3,0),(4,0), \ldots$ and $(0,2),(0,3),(0,4), \ldots$, but not for pairs $\left(n_{1}, n_{2}\right)$ for which both $n_{1}$ and $n_{2}$ are nonzero. In systems for which 
signals from the two bars interact in a nonlinear fashion, significant responses would be expected to be observed for $\left(n_{1}, n_{2}\right)$ pairs for which both $n_{1}$ and $n_{2}$ are nonzero, such as $\left(n_{1}, n_{2}\right)=(1,1)$ and $(1,-1)$.

\section{Results}

VEP responses elicited by two-frequency modulation of a Vernier target

We first consider the pattern of VEP components elicited by twofrequency modulation of Vernier targets in which the displacement $d$ was varied over a range that included alignment $(d=0)$, hyperacuity separations (0.5, and $1 \mathrm{~min})$, and larger separations (2, 4, and $8 \mathrm{~min}$ ). For this experiment, two frequency pairs (marked by
“*” in Fig. 2) were used: a pair of closely spaced frequencies $\left(f_{1}, f_{2}\right)=(3.027,3.223) \mathrm{Hz}$, and a pair of higher and more wellseparated frequencies $\left(f_{1}, f_{2}\right)=(4.980,7.129)$. For the first frequency pair, responses at the modulation frequencies and their second harmonics are plotted as vectors in the complex plane in Fig. 3 for one representative subject. All responses are significantly different from zero [that is, the $95 \%$ confidence limits as determined by the $T_{\text {circ }}^{2}$ statistic (Victor \& Mast, 1991)] do not include the origin. Moreover, for each frequency considered $\left[f_{1}\right.$ (A), $2 f_{1}(\mathrm{~B}), f_{2}(\mathrm{C})$, and $\left.2 f_{2}(\mathrm{D})\right]$, the responses do not have any consistent dependence on the Vernier displacement $d$. That is, the trajectory formed by the responses does not move systematically within the complex plane, and their error circles overlap extensively. A similar pattern was observed for stimuli modulated by other frequency pairs, and also with narrow bars. This lack of

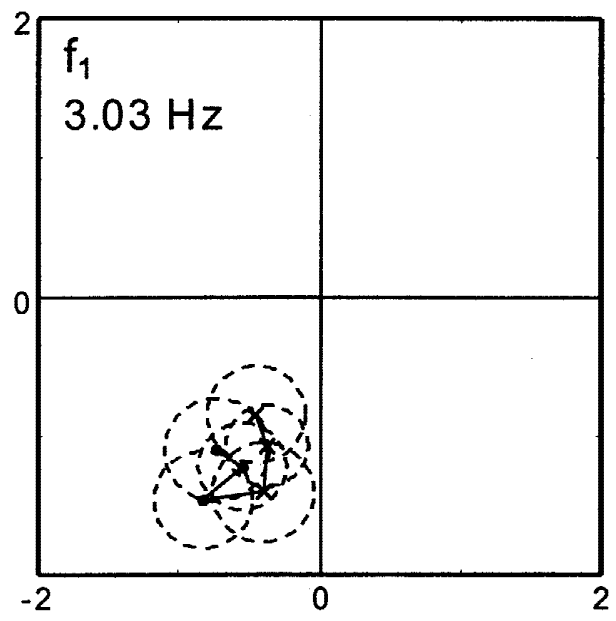

A.

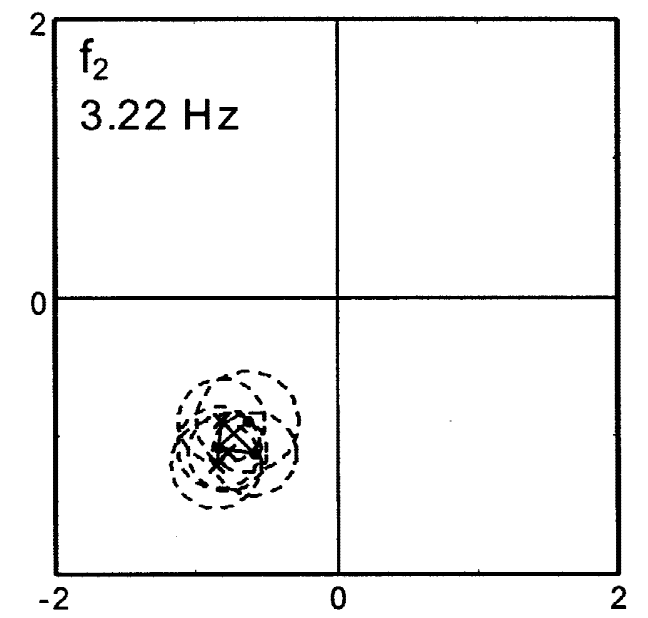

C.

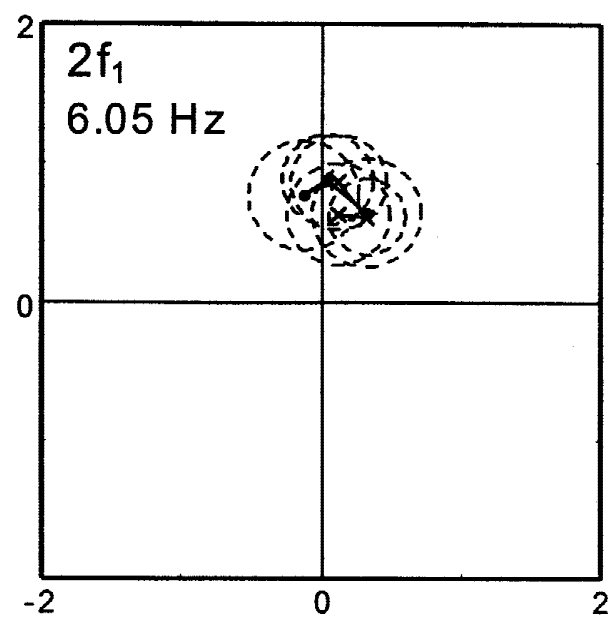

B.

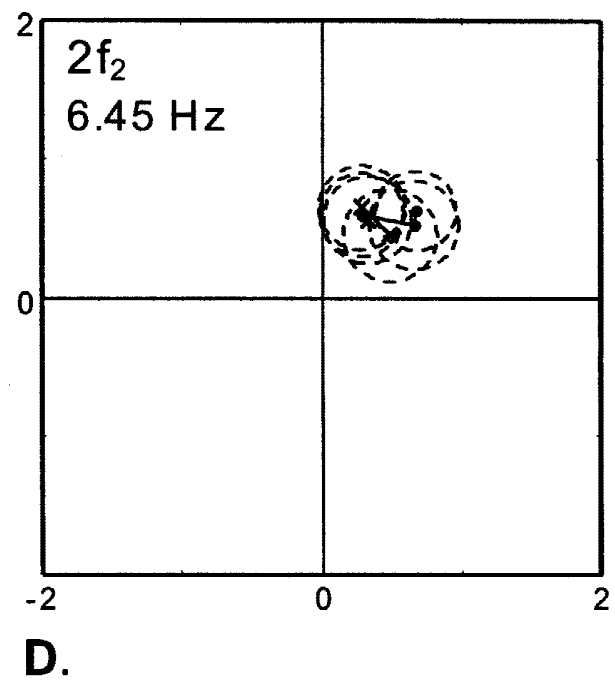

Fig. 3. Fourier components of VEP responses at the frequencies $f_{1}(\mathrm{~A}), 2 f_{1}(\mathrm{~B}), f_{2}(\mathrm{C})$, and $2 f_{2}$ (D), obtained with wide (7.5 min) bars and $\left(f_{1}, f_{2}\right)=(3.027,3.223) \mathrm{Hz}$. Fourier components of responses are plotted as points in the complex plane. The distance of a point from the origin represents the magnitude of the Fourier component, and the direction (counterclockwise from the positive real axis) of the vector from the origin to the point represents the phase of the response. The circle surrounding each point represents the $95 \%$ confidence limit for the response estimate, as determined by the $T_{\text {circ }}^{2}$ statistic (Victor \& Mast, 1991). Each panel shows the measured response component at a range of Vernier displacements $d$. Points marked by " $X$ " correspond to the three smaller displacements $(0$, 0.5 , and $1 \mathrm{~min})$; points marked by " $\bullet$ " corresponding to the three larger displacements (2, 4, and $8 \mathrm{~min})$. S: JV. 
dependence of the fundamental and second harmonic VEP components on Vernier displacement is not surprising, since these response components are primarily generated by the individual components of the Vernier targets, and not by their interactions.

A contrasting pattern is seen in the responses at the interaction frequencies (Fig. 4). With either frequency pair $\left(f_{1}, f_{2}\right)=$ $(3.027,3.223)$ or $(4.980,7.129)$, responses at $f_{1}+f_{2}[A(1,1)$ of eqn. (2)] showed a systematic dependence on displacement (panels A and B). For the three smaller displacements (points marked by " $\times$ ": $0,0.5$, and $1 \mathrm{~min}$ ), the error circles overlap extensively. For the three larger displacements (points marked by "๑": 2, 4, and $8 \mathrm{~min}$ ), there is a systematic movement of the response locus towards the origin. This dependence is beyond the intrinsic variability of the responses, as indicated by the separation of the error circles. Note that the origin corresponds to a null Fou- rier component, that is, the absence of a VEP indicator of an interaction between the bars.

A similar systematic dependence is seen for responses at the difference frequency $f_{1}-f_{2}[A(1,-1)$ of eqn. (2)] for the frequency pair $\left(f_{1}, f_{2}\right)=(4.980,7.129)$ (panel D), but not for the difference frequency responses elicited by the frequency pair $\left(f_{1}, f_{2}\right)=(3.027,3.223)$ ( panel $\left.\mathrm{C}\right)$. The response variability, as indicated by the size of the error circles, is also much larger in the latter dataset. Background EEG power represents the main source of response variability (Mast \& Victor, 1991; Victor \& Mast, 1991), and thus the response uncertainties are much larger at low frequencies $\left(\left|f_{1}-f_{2}\right|=0.195 \mathrm{~Hz}\right.$ in panel $\left.\mathrm{C}\right)$ than at high frequencies at $\left(\left|f_{1}-f_{2}\right|=2.148 \mathrm{~Hz}\right.$ in panel $\left.\mathrm{D}\right)$. That is, the inability to discern significant responses at $f_{1}-f_{2}=0.195 \mathrm{~Hz}$ in panel $\mathrm{C}$ is not due to the fact that it is a difference frequency per se, but merely

\section{Bar Size $=7.5 \times 60 \mathrm{~min} \quad$ Bar Size $=1.7 \times 60 \mathrm{~min}$}
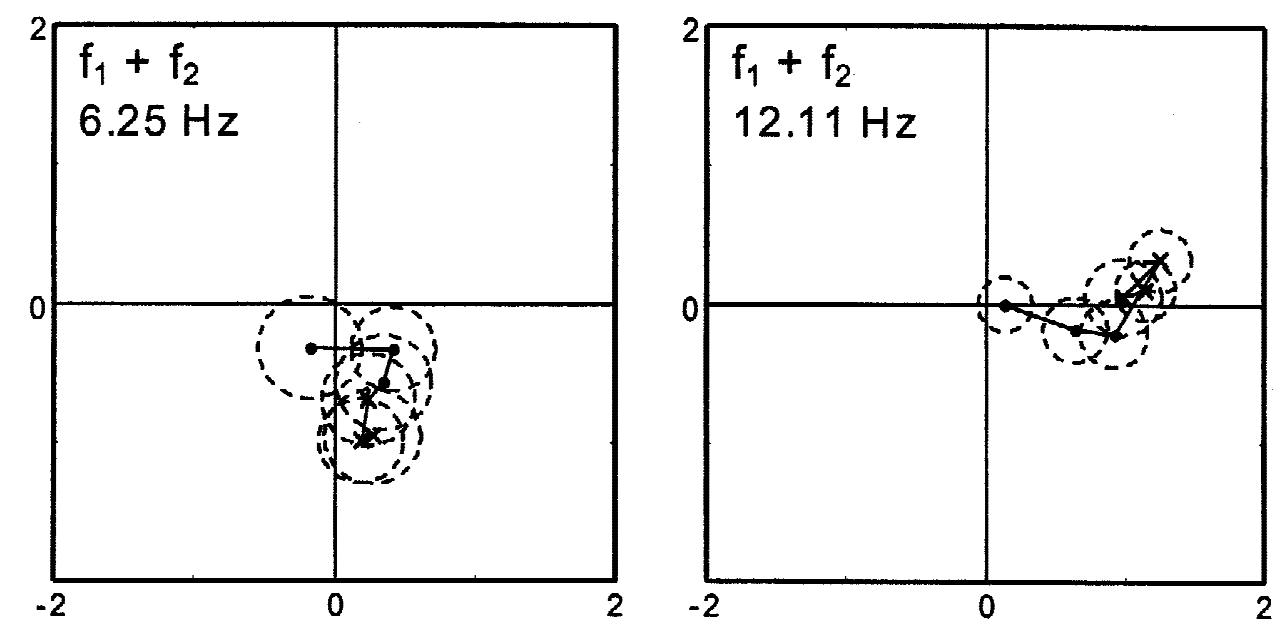

A.

B.
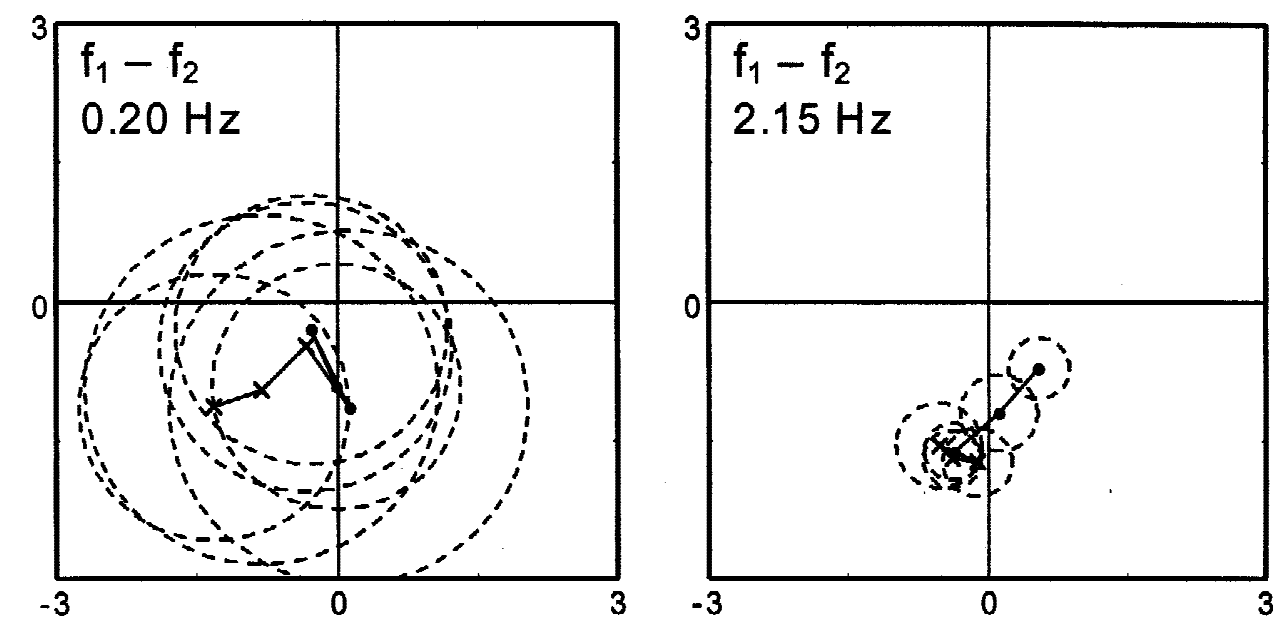

C.

D.

Fig. 4. Fourier components of VEP responses at the interaction frequencies $f_{1}+f_{2}(\mathrm{~A}, \mathrm{~B})$ and $f_{1}-f_{2}(\mathrm{C}, \mathrm{D})$, obtained with wide $(7.5 \mathrm{~min})$ bars $(\mathrm{A}, \mathrm{C})$ and $\left(f_{1}, f_{2}\right)=(3.027,3.223) \mathrm{Hz}$, or narrow $(1.7 \mathrm{~min})$ bars $(\mathrm{B}, \mathrm{D})$ and $\left(f_{1}, f_{2}\right)=(4.980,7.129) \mathrm{Hz}$, at each of six Vernier displacements $d$. Responses and confidence limits are plotted as in Fig. 3. S: JV. 
because it lies in an unfavorable portion of the EEG spectrum (see Fig. 12). For this reason, we will focus on the interaction at the sum frequency $f_{1}+f_{2}$ (in this case, 6.25 and $12.11 \mathrm{~Hz}$ ), where response uncertainties were small for all of the input frequency pairs and across subjects.

Fourier components of another subject's responses at the sum frequency $f_{1}+f_{2}$ are shown in Fig. 5, for the two frequency pairs and both bar widths. In all cases, the response changes systematically as a function of Vernier displacement $d$, as indicated by the arcuate response trajectory of the responses in the complex plane. As in the data of Figs. 4A, 4B, and 4D, the error circles overlap for the small displacements, and only become statistically distinguishable for displacements of 2, 4, and 8 min (points marked by "๑”). Comparison of responses at the two frequency pairs (A vs. C, B vs. D) reveals an approximate rotation of the response locus in the complex plane, of approximately one quarter of a cycle counter- clockwise. This pattern of rotation is consistent with (but not as clearly seen in) the data of Fig. 4 (A vs. B), and suggests that the frequency dependence of the responses can be explained by an effective latency. This will be examined more extensively below. Fig. 5 also shows (A vs. B, C vs. D) that the overall size and frequency dependence of the responses are relatively independent of bar width.

In Figs. 4A and 4B, the error circle around the response at the largest Vernier displacement ( $8 \mathrm{~min}$ ) includes the origin, indicating that this interaction response is not significantly different from zero when the components of the Vernier target are maximally separated. However, this behavior is not seen in all subjects. In particular, in the data of Fig. 5, the smallest interaction responses are seen when the Vernier displacement $d$ is 4 min. (In Fig. 5B, the 2-min response is smaller than the 4-min response, but the difference is not statistically significant.) Presumably, the VEP evidence

\section{Bar Size $=7.5 \times 60 \mathrm{~min} \quad$ Bar Size $=1.7 \times 60 \mathrm{~min}$}

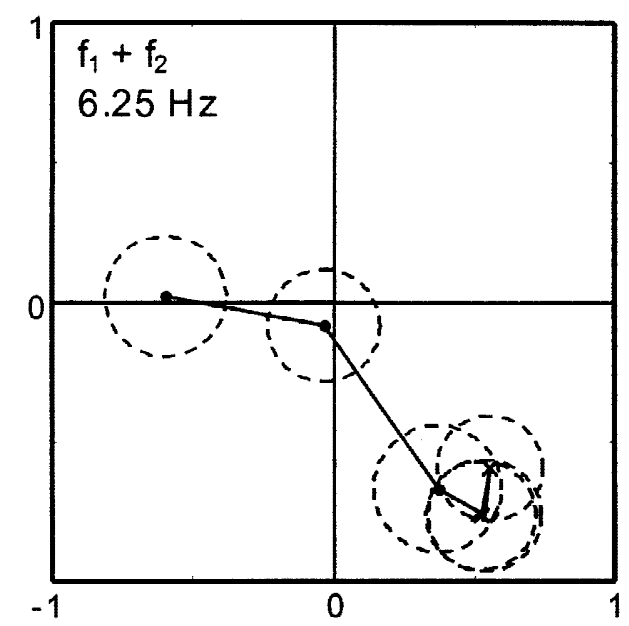

A.

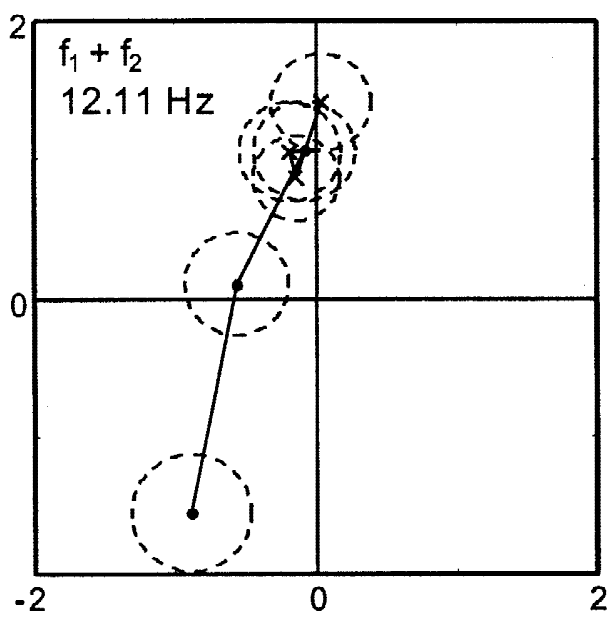

C.

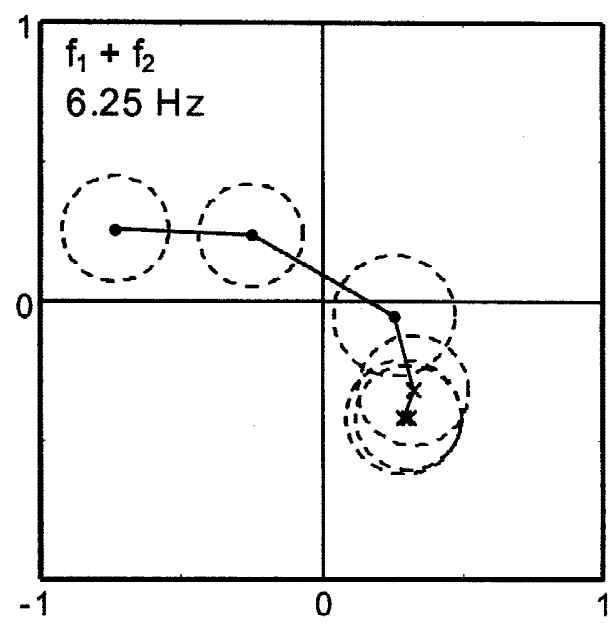

B.

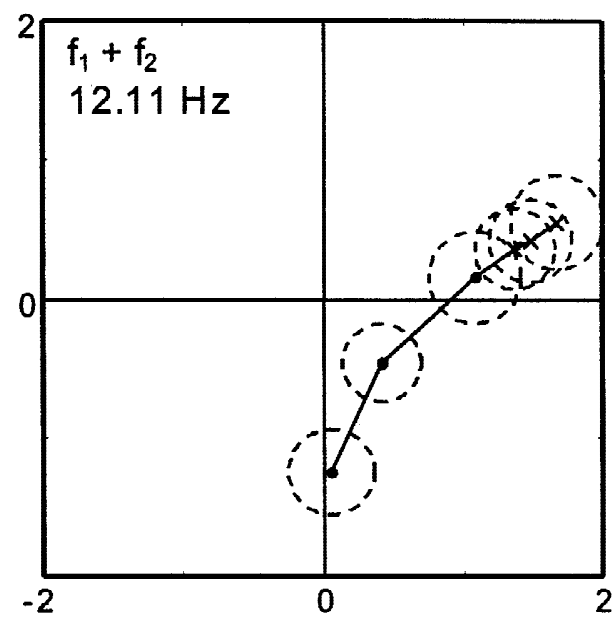

D.

Fig. 5. Fourier components of VEP responses at the intermodulation frequency $f_{1}+f_{2}$, for wide ( $7.5 \mathrm{~min}$ ) bars (panels A, C) and narrow (1.7 min) bars (panels B, D), and two frequency pairs: $\left(f_{1}, f_{2}\right)=(3.027,3.223) \mathrm{Hz}$ (panels A, B) and $\left(f_{1}, f_{2}\right)=(4.980,7.129) \mathrm{Hz}$ (panels C, D), at each of six Vernier displacements $d$. Responses and confidence limits are plotted as in Fig. 3. S: MC 
of interactions recorded at large displacements reflect the fact that bars modulated at $f_{1}$ in one Vernier target can interact with bars modulated at $f_{2}$ in another target. Increasing the separation of bars within a target necessarily decreases the separation of bars in adjacent targets.

Another prominent feature of Figs. 4 and 5 is that the trajectories are consistently curved. If there were only one displacementdependent interaction (that is, an interaction whose strength but not timing depended on the distance $d$ ), then the response trajectory would be a straight line. The direction of this line would correspond to the phase of this putative solitary interaction, and the movement of the trajectory along this line would correspond to recruitment of more or less of this interaction, parametric in $d$. Conversely, the curvature of the trajectories implies that the displacement-dependent component of the interaction is not fixed in latency, or, that distinct interactions (of different latencies) are recruited at different displacements. The direction of the curvature (counterclockwise with increasing displacement) implies that the effective latency of this interaction is earlier (more phase lead) with increasing displacement $d$. This is opposite to the behavior characteristic of a propagation delay, which would be expected to show more phase lag with increasing displacement $d$. That is, the counterclockwise curvature suggests a combination of two (or more) sources of interaction: a short-latency, or fast, interaction that dominates at large displacements, and a long-latency, or slow, interaction that dominates at small displacements but may well be present at all displacements.

Because these features of the data were found for both bar widths, they depend on the Vernier displacement $d$, rather than on the extent of overlap at the abutting ends of the bars. To assess the dependence on $d$ rigorously, we used an analysis of variance adapted for complex-valued quantities (the Fourier components). For each subject, we compared the variability within trials at the same displacement $d$ (the areas of the error circles) with the variability across trials with different values of the displacement (the squared distances of the error circles from their mean). This was broken down into a comparison for the three stimuli within the hyperacuity range $(d=0,0.5$, and $1 \mathrm{~min})$ and the three larger displacements ( $d=2,4$, and $8 \mathrm{~min})$, and was carried out for each subject $(N=$ 5 ) and for each of the 30 interaction frequencies up to order 6 . The statistical summary (Fig. 6) was pooled across bar widths, given the similarity of responses as shown in Fig. 5. For displacements within the hyperacuity range, the number of response components for which there was a detectable dependence on offset was not different from chance expectation (5\% false-alarm rate). However, for larger displacements, a statistically significant fraction of the responses showed a dependence on the displacement $d$. This was consistent across frequency pairs and also held for analyses restricted to each bar width and within each subject. In none of the five subjects were there a significant fraction of responses that depended on $d$ within the hyperacuity range, while in four of the five subjects (all but EM), a significant fraction of the responses did depend on $d$ for the larger displacements. Note that the comparison between the hyperacuity range analysis and the largerdisplacement analysis is equated for the number of degrees of freedom, and thus for statistical power.

Even for the larger displacements, the responses that showed a statistically significant dependence on displacement were concentrated in the lower-order components (see also Fig. 8 below), and particularly at the sum frequency $f_{1}+f_{2}$. When the above analysis is restricted to this frequency alone, 13 of the 20 comparisons (five subjects, two frequency pairs, two bar widths) showed a significant

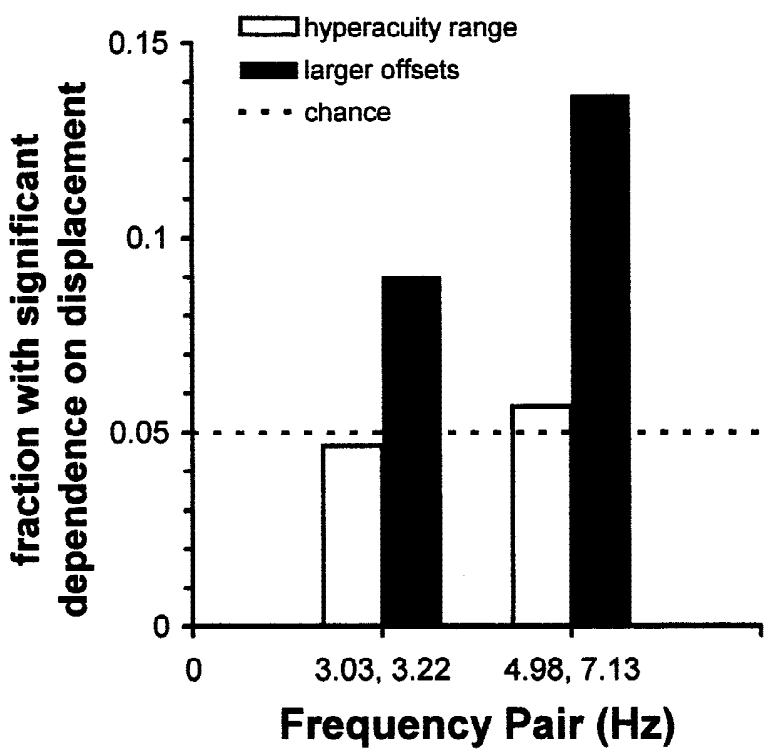

Fig. 6. The fraction of responses that had a significant dependence on Vernier displacements, as determined by the $T_{\text {circ }}^{2}$ statistic. Dependence on Vernier displacement is assessed across the three smaller displacements $d(0,0.5$, or $1 \mathrm{~min})$, open bars, and across the three larger displacements $d$ $(2,4$, or $8 \mathrm{~min})$, filled bars, as detailed in the text. A $5 \%$ criterion was used for statistical significance. For each frequency pair (“*” in Fig. 2), data are pooled across subjects, both bar sizes, and interaction frequencies up to order 6.

dependence on displacement in the larger range, but only two of the 20 comparisons (chance expectation: one comparison) showed a significant dependence on displacement in the hyperacuity range. Thus, although there is a clear VEP signature of interactions between the bars that depend on their spatial separation, we did not identify interaction components that were specific to the hyperacuity range.

\section{The dynamics of the VEP interaction components}

The dynamics of the responses at the sum frequency $f_{1}+f_{2}$ were studied more intensively in a second experiment, in which five frequency pairs (those connected by a line in Fig. 2) were used. For these frequency pairs, sum frequencies ranged from $6.25 \mathrm{~Hz}$ to $14.06 \mathrm{~Hz}$ in approximately $2-\mathrm{Hz}$ steps. Vernier offsets were restricted to 0,1 , and $8 \mathrm{~min}$, and only the narrow bar width was used. Response amplitude and phase for two subjects at $f_{1}+f_{2}$ are shown in Fig. 7. In agreement with the previous experiment, there were no consistent differences between the interaction component elicited by the aligned Vernier target and the target with a displacement of $1 \mathrm{~min}$, but the interaction component elicited by the target with the large displacement was generally smaller.

Within each condition, the response amplitudes depend only modestly on temporal frequency, declining by not more than a factor of two from 6 to $14 \mathrm{~Hz}$. Moreover, the response phases change approximately linearly, and thus the slope of the phase curve can be considered to be an effective latency. These latencies are presented in Table 1 for the five subjects. For the aligned condition and the hyperacuity range displacement, the latencies cluster relatively tightly around 145 to $165 \mathrm{~ms}$, and the variability 

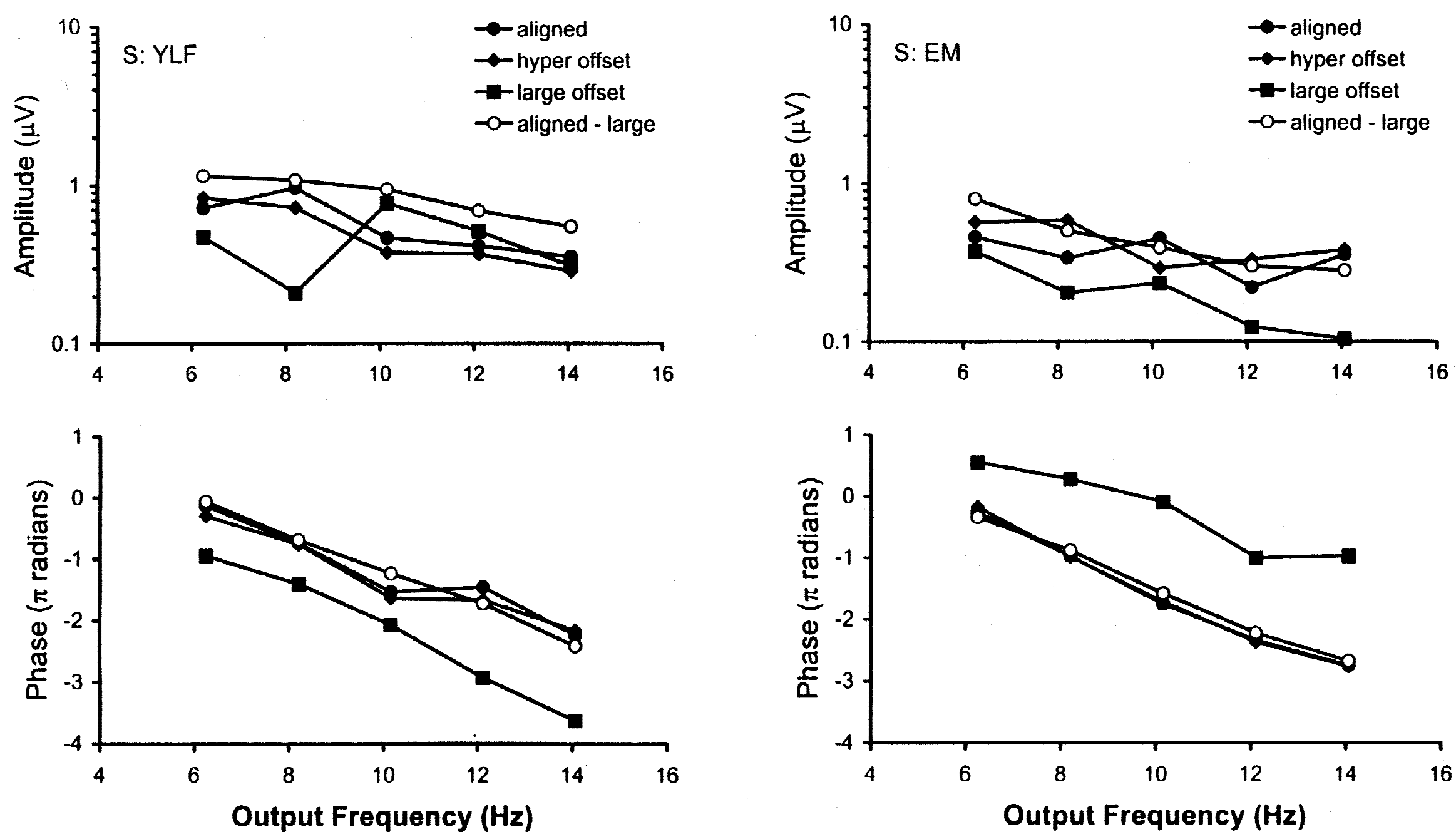

Fig. 7. Response amplitude and phase at the sum frequency $f_{1}+f_{2}$ for Vernier targets in which bars were aligned $(\bullet)$, a hyperacuity-range offset of 1 min $(\bullet)$, and a larger offset of $8 \mathrm{~min}(\boldsymbol{\square})$ for two subjects. The five frequency pairs used are those connected by the lines in Fig. 2. The curve for "aligned-large" $(\mathrm{O})$ is calculated by a vector subtraction of the measured responses. 
Table 1. Effective latencies of the Fourier component at the sum frequency $f_{1}+f_{2}$ for Vernier targets in which bars were aligned, separated by a hyperacuity-range displacement of $1 \mathrm{~min}$, or by a larger displacement of $8 \mathrm{~min}^{\mathrm{a}}$

\begin{tabular}{lcccccc}
\hline \hline & \multicolumn{5}{c}{$f_{1}+f_{2}$ Response Latencies (ms) } \\
\cline { 2 - 7 } & MC & JV & YLF & JT & EM & Average \\
\hline aligned & 147 & 155 & 126 & 166 & 163 & 151 \\
hyper & 147 & 149 & 119 & 160 & 168 & 149 \\
large & 126 & 194 & 176 & 110 & 111 & 143 \\
aligned-large & 139 & 134 & 147 & 124 & 154 & 140 \\
& & & & & & \\
\hline \hline
\end{tabular}

${ }^{a}$ These latencies were determined by the slope of the best-fitting line to the phase curves in Fig. 7 for subjects YLF and EM, and for comparable data (not shown) from the other three subjects. The "aligned-large" latencies were determined from the slope of the phases of the vector differences of the measured responses.

is primarily across subjects $[F(9,4)=44.1, P=0.0014]$, rather than between conditions ( $P=0.35$, two-tailed paired $t$ test).

There is no consistent difference between the latencies of the large-offset responses and those measured for the aligned condition $(P=0.74$, two-tailed paired $t$ test), but the former latencies do show a larger intersubject variability. This variability most likely reflects variation in the relative sizes of multiple contributions to the interactions, as discussed above in connection with Fig. 4 $(\mathrm{A}, \mathrm{B})$ and Fig. 5. We removed the displacement-independent contribution to the large-offset responses by vector subtraction of the responses measured in the "aligned" condition ("aligned-large" in Table 1). Latencies of this residual displacement-dependent contribution have less intersubject variability, and remain similar to the latencies of the displacement-dependent component(s) observed in the "aligned" condition.

\section{Higher-order interactions}

We now consider the Fourier components of the responses at all harmonics and intermodulation frequencies (42 frequencies up to order 6), not restricting consideration to the responses with a demonstrable dependence on displacement $d$. Fig. 8 shows the fraction of the responses $A\left(n_{1}, n_{2}\right)$ [eqn. (2)] that were significantly different from zero for each harmonic order $\left(\left|n_{1}\right|+\left|n_{2}\right|\right)$, as determined by the $T_{\text {circ }}^{2}$ statistic (Victor \& Mast, 1991). Data are pooled across the five subjects and across the six bar displacements, so that a total of 30 assessments were made for each possible response frequency, and 5\% of the responses were expected to be statistically "significant" by chance alone. (We summarize the response spectrum in terms of fraction of significant responses, rather than the size of the responses, since response amplitude is likely to be influenced by the dynamics underlying EEG generation, and thus would be expected to be smaller for higher response frequencies $n_{1} f_{1}+n_{2} f_{2}$. Examination of response significance, rather than overall response size, compares the observed response to the EEG background. Thus, to a first approximation, this response normalization removes the potential confound of EEG dynamics on the assessment of higher-order responses).

As seen in Fig. 8, the pattern of significant responses is largely independent of bar width and the frequency pair chosen. The majority of responses at first-order frequencies $[A(1,0)$ and $A(0,1)$ of eqn. (2), the responses at $f_{1}$ and $f_{2}$ ] were significantly different from zero. The same is true for the second-order frequencies, for both the pure second-harmonic responses $[A(2,0)$ and $A(0,2)$ of eqn. (2), the responses at $2 f_{1}$ and $\left.2 f_{2}\right]$ and the intermodulation responses $[A(1,1)$ and $A(1,-1)$ of eqn. (2), the responses at $f_{1}+f_{2}$ and $\left.f_{1}-f_{2}\right]$. At higher harmonics, the fraction of significant responses does not decline monotonically with the harmonic order. Rather, the fourthorder responses are more prominent than either the third- or fifthorder responses. Fifth- and sixth-order responses were detected at a rate only marginally above chance, and not in all subjects.

It is useful to classify the responses $A\left(n_{1}, n_{2}\right)$ by the parity of $n_{1}$ and $n_{2}$ (Regan \& Regan, 1988b; Regan, 1990). For example, the responses $A(1,1)$ and $A(1,-1)$ may be generated by multiplicative interactions from signals derived from the two inputs by a linear filtering process. However, if a full-wave rectifier precedes the interaction of the two inputs, then only responses $A\left(n_{1}, n_{2}\right)$ for which both $n_{1}$ and $n_{2}$ are even may arise. If the interaction between the two inputs is not strictly multiplicative, responses $A\left(n_{1}, n_{2}\right)$ for which $n_{1}$ and $n_{2}$ are odd may arise, but only from interactions of the unrectified portions of the two inputs. For this reason, a comparison of response components with even and odd parities of $n_{1}$ and $n_{2}$ can suggest whether there is rectification that precedes the interaction of the inputs. But to make this comparison meaningful, it is necessary to eliminate consideration of responses $A\left(n_{1}, n_{2}\right)$ for which either $n_{1}$ and $n_{2}$ is zero, since these responses can arise from nonlinear transformation of a single input in isolation, and thus need not reflect interactions. Thus, the simplest informative comparison concerns fourth-order frequencies, namely, a comparison of the fraction of significant even-parity mixed responses $[A(2,2)$ and $A(2,-2)]$ with the fraction of significant odd-parity mixed responses $[A(3,1), A(1,3), A(3,-1)$, and $A(1,-3)]$. As seen in Fig. 9, the responses at the even-parity mixed responses were more prominent than the responses at the odd-parity mixed responses. This was seen not only in the data pooled across subjects, but in analyses of each of the individual subjects' data as well, for both frequency pairs and bar widths.

Superimposed on this overall finding were two aspects that depended on the choice of frequency pairs. The even-parity mixed responses were less prominent for the nearly-matched frequency pair $(3.027,3.223)$ than for the other frequency pair $(4.980,7.129)$. This reflected an inability to detect significant $A(2,-2)$ responses for the nearly-matched frequency pair, since the resulting output frequency $2 f_{1}-2 f_{2}$ is low $(0.391 \mathrm{~Hz})$, within the range in which intrinsic response variability is high (see also Fig. 4C). The second trend was that the pure fourth-order responses were less prominent for the high-frequency pair $(4.980,7.129)$ than for the lowfrequency pair. This primarily reflected a lack of significance of responses $A(0,4)$, whose output frequency $(28.515 \mathrm{~Hz})$ was much higher than any of the fourth-order frequencies in the nearlymatched frequency pair.

\section{Dependence of VEP interaction components on gap}

Next, we examined the VEP interaction responses elicited by measuring responses to targets in which the bars remained aligned, but were separated by small horizontal gaps $g(1,2,4$, and $8 \mathrm{~min})$, rather than the vertical displacements studied above.

As shown in the representative vector plots of Fig. 10 (trajectory with filled symbols), gap-dependent interactions were seen at the combination frequencies $f_{1}+f_{2}$ (panel A) and $f_{1}-f_{2}$ (panel B). The trajectories moved in a similar, though not identical, direction as the trajectories associated with changing Vernier displacement $d$ 
Bar Size: 1.7 x $60 \mathrm{~min}$

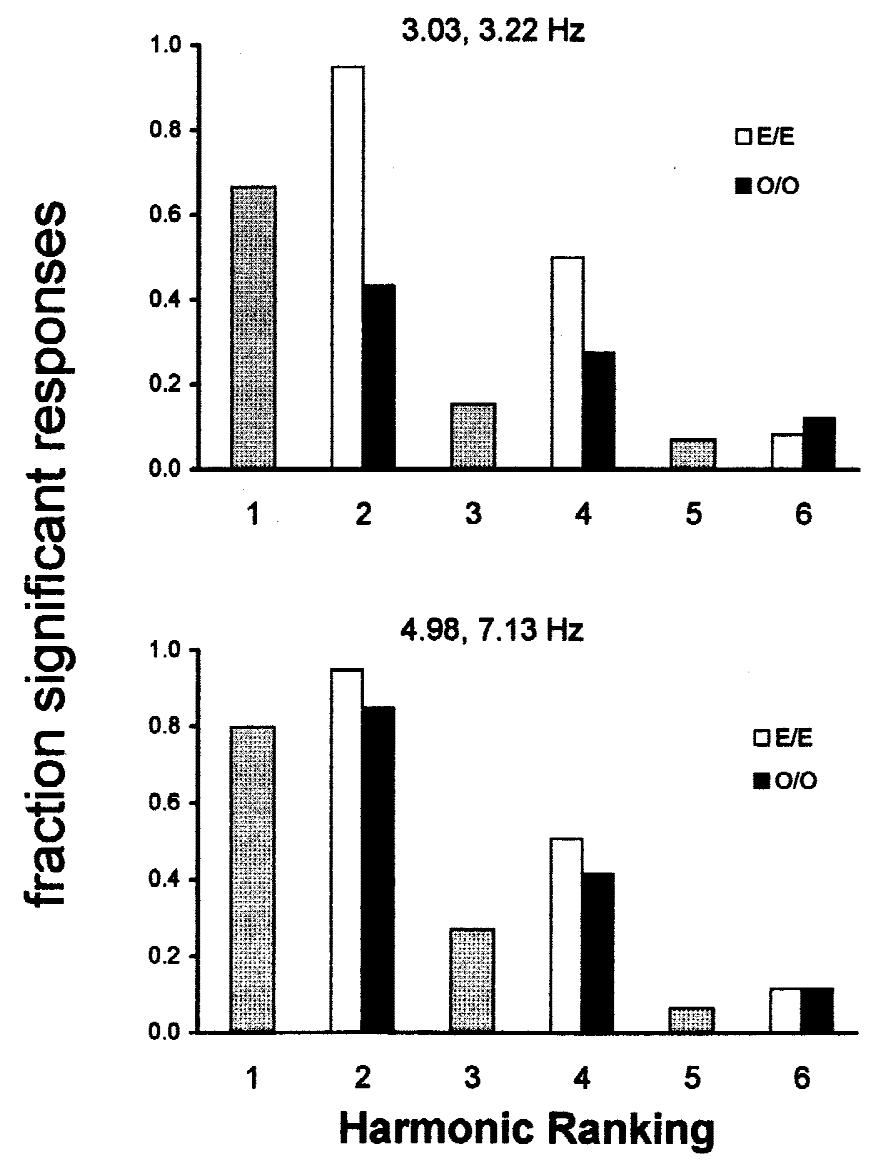

\section{Bar Size: 7.5 x $60 \mathrm{~min}$}
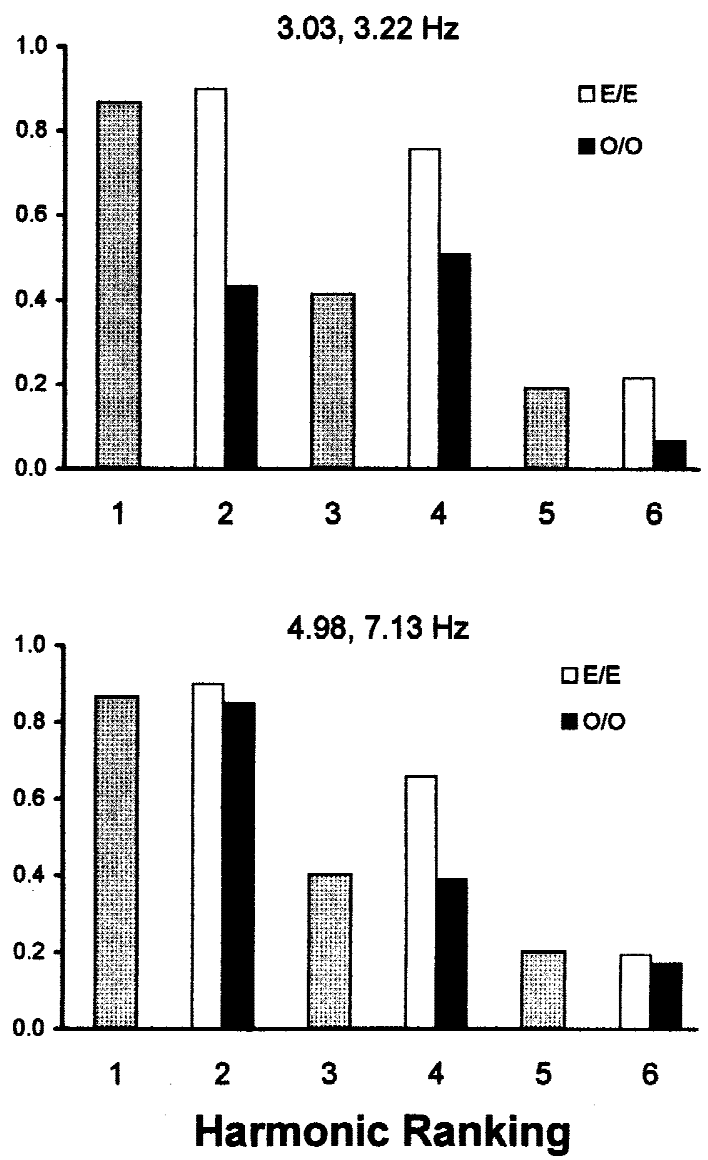

Fig. 8. The fraction of responses that were significantly different from 0 , as determined by the $T_{\text {circ }}^{2}$ statistic, as a function of response order. For even-order responses $\left(n_{1} f_{1}+n_{2} f_{2}\right.$ for which $n_{1}+n_{2}$ is even), the analysis is subdivided according to whether both $n_{1}$ and $n_{2}$ are even (open bars), or both are odd (filled bars). A 5\% criterion was used for statistical significance. Data pooled across all six values of the displacement $d$ and subjects, and shown separately for the two bar widths and two frequency pairs (“*” in Fig. 2).
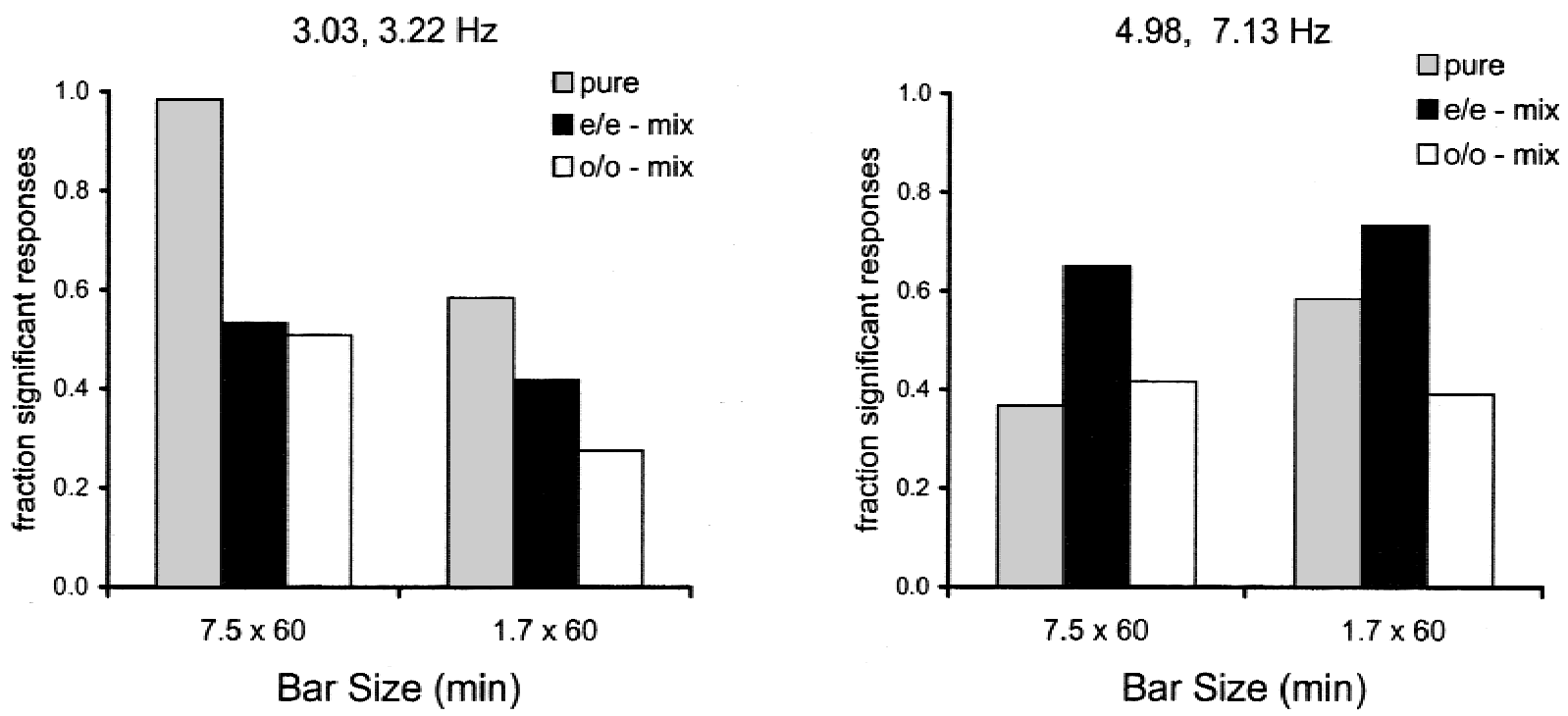

Fig. 9. Further analysis of the fourth-order responses of Fig. 8. Fourth-order response frequencies $n_{1} f_{1}+n_{2} f_{2}$ are subdivided into three categories: pure fourth-order responses ( $4 f_{1}$ or $4 f_{2}$, gray bars), mixed even-order ( $2 f_{1} \pm 2 f_{2}$, open bars), and mixed odd-order ( $3 f_{1} \pm$ $f_{2}$ and $f_{1} \pm 3 f_{2}$, filled bars). 


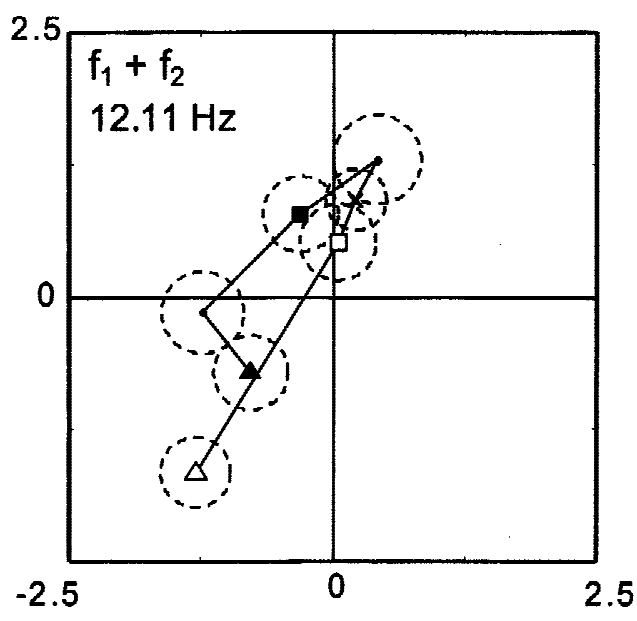

A.

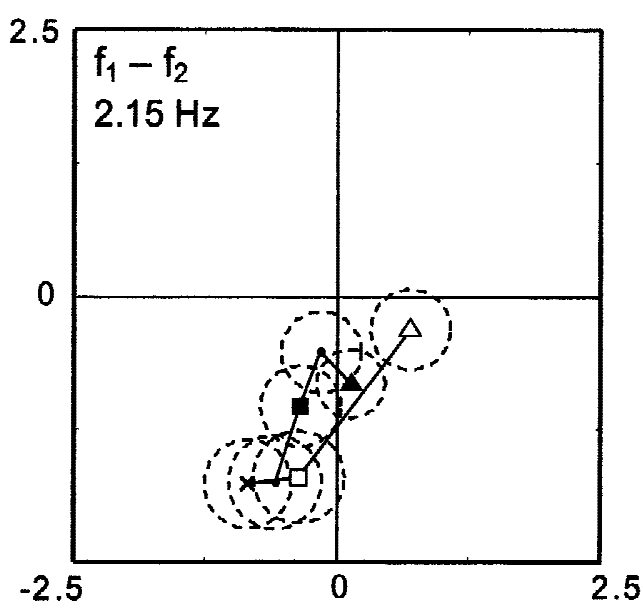

B.

Fig. 10. Comparison of dependence of the $f_{1}+f_{2}$ (panel A) and $f_{1}-f_{2}$ (panel B) Fourier components of VEP response on Vernier displacement $d$ ( 2 and $8 \mathrm{~min}$, trajectories with open symbols) and gap $g(1,2,4$, and $8 \mathrm{~min}$, trajectories with filled symbols). Stimuli consisted of narrow $(1.7 \mathrm{~min})$ bars modulated at the frequency pair $\left(f_{1}, f_{2}\right)=(4.980,7.129) \mathrm{Hz}$. Responses for aligned, abutting bars $(d=0, g=0)$ are marked by "X". Responses to the stimuli with displacement or gap of 2 min are marked by squares; responses to stimuli with displacement or gap of 8 min are marked by triangles. Confidence limits are plotted as in Fig. 3. S: MC.

(open symbols). For large positional changes, the VEP was more sensitive to a change in Vernier displacement than to a change in gap ( $8 \mathrm{~min}$, triangles). But for small positional changes ( $2 \mathrm{~min}$, squares, there was no difference in sensitivity at $f_{1}+f_{2}$ (panel A), and at $f_{1}-f_{2}$ (panel B), the relative sensitivity was reversed. That is, a 2-min gap (marked by " $\square$ ") produced a response whose error circle did not overlap with the error circle around the response elicited by aligned, abutting bars $(d=0, g=0$, marked by " $\times$ "), while the error circle around the response elicited by the 2-min displacement (marked by " $\square$ ") overlapped significantly with the error circle around the " $\times$ ".

Fig. 11 summarizes the pattern of VEP sensitivity to horizontal displacements. For the closely separated frequency pair (left), only the larger displacements led to a reliable change in the VEP. For the widely separated frequency pair, both small and large gaps produced a significant change in the VEP (right). The presence of VEP components sensitive to small horizontal displacements was sufficiently robust so that it could be seen in three of the five subjects' individual data (MC, JT, JV) for the widely separated frequency pair, and in one subject's data (MC) for the closely separated frequency pair. This contrasts with the data of Fig. 6, which showed no consistent dependence of the interaction components for Vernier displacements within the hyperacuity range for either frequency pair, in any of the five subjects.

\section{Undriven components}

Thus far, we have assessed the response to the two-frequency stimuli by considering the average Fourier components elicited at $n_{1} f_{1}+n_{2} f_{2}$, namely, $A\left(n_{1}, n_{2}\right)$ [eqn. (2)]. Period-to-period variability in the estimates of these estimated Fourier components $\left[A_{m}\left(n_{1}, n_{2}\right)\right.$, eqn. (1)] was considered to be "noise" and used to assess the reliability of the measurements of $A\left(n_{1}, n_{2}\right)$, but was not considered to be a response measure itself. This corresponds to the notion that "signal" $\left[A\left(n_{1}, n_{2}\right)\right]$ adds linearly to the ongoing EEG and does not interact with it, and thus variability in estimates of $A\left(n_{1}, n_{2}\right)$ reflects the power spectrum of the background EEG
(Mast \& Victor, 1991). However, this is only an approximation, and driven components of an event-related response may interact with ongoing EEG (Basar, 1980; Mast \& Victor, 1991; TallonBaudry et al., 1996, Pfurtscheller \& Andrew, 1999).

One way of assessing a possible interaction between the driven response and the EEG background is to measure the power spectrum of the EEG, with the driven response removed (Mast \& Victor, 1991). These are the quantities $P\left(n_{1}, n_{2}\right)$ of eqn. (3). Under

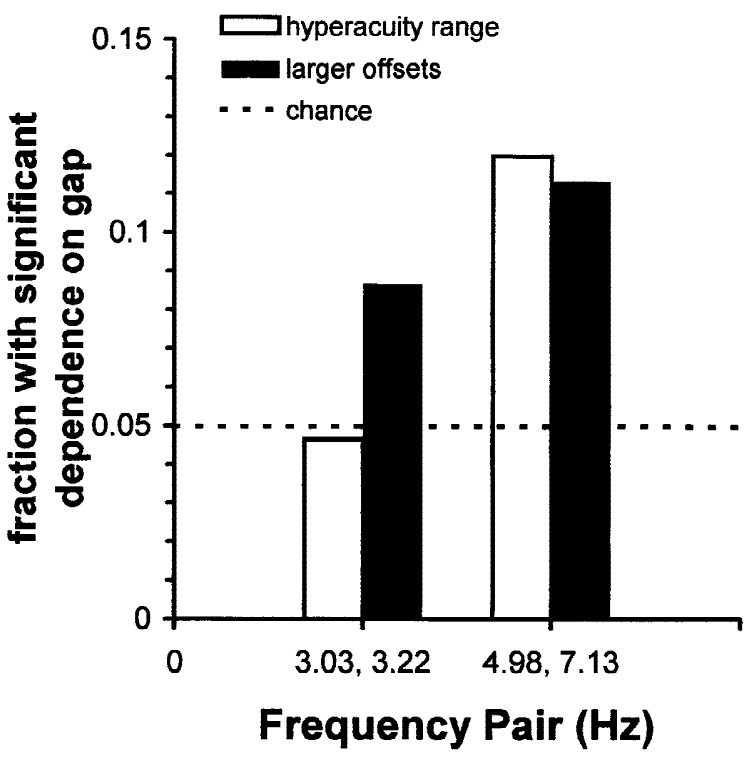

Fig. 11. The fraction of responses that had a significant dependence on gap $g$. Significant dependence is separately assessed for the smaller displacement $(0,1$, and $2 \mathrm{~min})$ and the larger displacement (2, 4, and $8 \mathrm{~min})$ by the $T_{\text {circ }}^{2}$ statistic. A 5\% criterion was used for statistical significance. Data pooled across all frequencies up to order 6 , and across all five subjects. Wide (7.5 min) bars. 
the assumption of independence of signal and noise, the power spectrum should be independent of the presence of the stimulus. In particular, the power spectrum should not have peaks associated with the stimulus frequencies or intermodulation frequencies at which a strong driven response is present.

We examined responses elicited by modulation of aligned bars by each of the nine frequency pairs (Fig. 2). Fig. 12 shows power spectra obtained in two subjects, with all data from each subject plotted on the same axes. For subject MC (top), there are modest peaks at the second harmonics of the input frequencies, as seen by the points (O) near 10 and $14 \mathrm{~Hz}$. This indicates synchronization of cortical activity at these frequencies, but not in a phase that is locked to that of the stimulus. There is also a peak at the sum frequency $f_{1}+f_{2}(*)$, indicating that the interaction between the two bars also drives cortical activity in a nonphase-locked manner. Order-by-order analysis (not shown) of the higher harmonics, with or without attention to the parity (even $v s$. odd) of the interactions, revealed no indications of elevation in $P\left(n_{1}, n_{2}\right)$ at the third- or higher-order frequencies, plotted uniformly as ( ). Analysis of variabilities $P\left(n_{1}, n_{2}\right)$ in subject JV (bottom) showed no evidence of peaks at the input frequencies or the intermodulation frequencies.

Fig. 13 shows the variabilities $P\left(n_{1}, n_{2}\right)$ measured in a separate experiment, in which responses at five frequency pairs were mea-
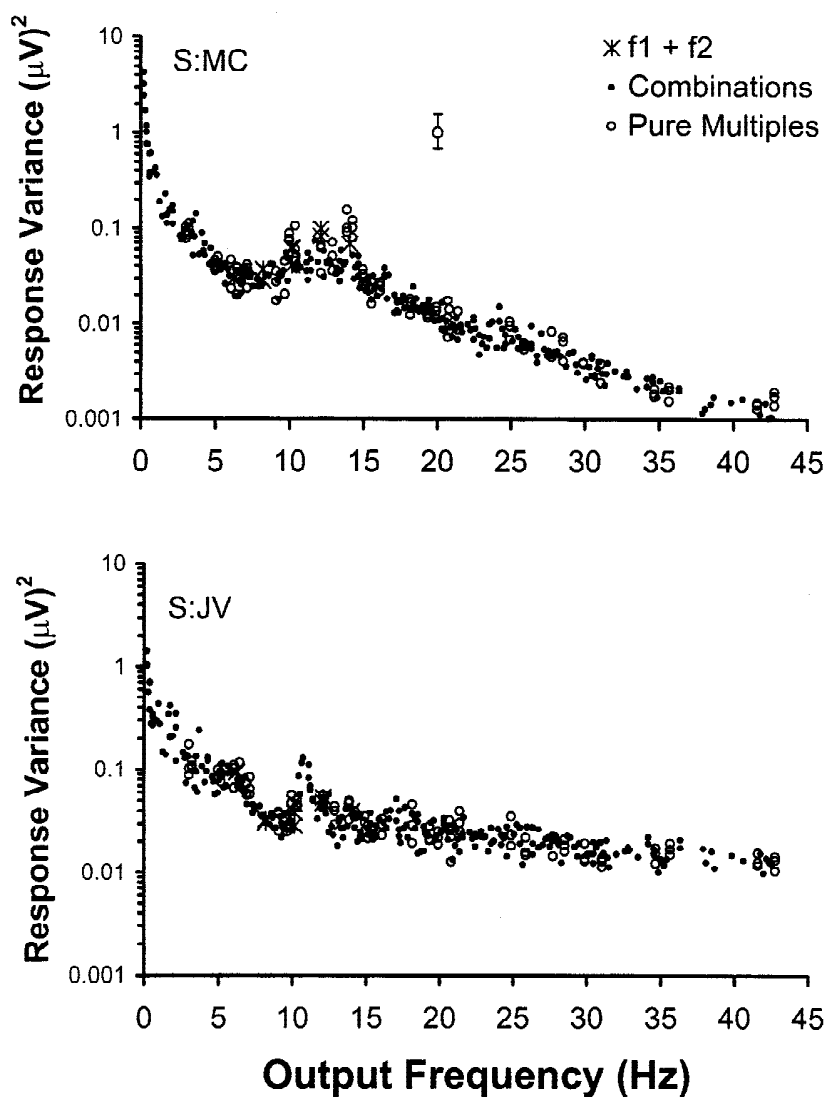

Fig. 12. Undriven activity elicited by the Vernier target, as assessed by response variability $\left[P\left(n_{1}, n_{2}\right)\right.$, eqn. (3)] for subject $\mathrm{MC}$ (top) and JV (bottom). Values of $P\left(n_{1}, n_{2}\right)$ are plotted as a function of the output frequency $n_{1} f_{1}+n_{2} f_{2}$, and data from all nine frequency pairs of Fig. 2 are superimposed. Pure harmonic multiples (O), sum frequency $f_{1}+f_{2}(*)$, other intermodulation frequencies up to order $6(\bullet)$. Displacement $d=0$. Wide $(7.5 \mathrm{~min})$ bars. The error bar (top panel) indicates the $95 \%$ confidence limit for all data, determined as described in Mast and Victor (1991). sured (those connected by a line in Fig. 2), at three Vernier displacements $(d=0,1$, and $8 \mathrm{~min})$. Most of the subjects (four out of five, three illustrated in Figs. 13A, 13C, and 13D) showed a modest enhancement of variability at the second harmonics of the input frequencies (points " $\mathrm{O}$ " in the 10 to $14 \mathrm{~Hz}$ range), as well as at the sum frequency $f_{1}+f_{2}(*)$. However, as seen in Fig. 13B, this pattern of enhanced background activity was not specific to the aligned condition, and was also seen with Vernier displacements.

\section{Discussion}

\section{Two-frequency analysis of a nonlinear interaction}

When two inputs of a nonlinear system are simultaneously driven by sinusoidal inputs at distinct frequencies $f_{1}$ and $f_{2}$, the response may contain Fourier components at various combination frequencies $n_{1} f_{1}+n_{2} f_{2}$. Responses at harmonics of the input frequencies, $n_{1} f_{1}$ and $n_{2} f_{2}$, can be generated by any nonlinearity. Responses at combination frequencies $n_{1} f_{1}+n_{2} f_{2}$ in which $n_{1}$ and $n_{2}$ are both nonzero can only be generated by elements that have access to both inputs, and in which these inputs interact nonlinearly (Regan $\&$ Regan, 1988b). With appropriate choice of visual stimuli and of the components to be sinusoidally modulated, the presence of such interaction frequencies in the VEP has identified lateral spatial interactions (Zemon \& Ratliff, 1984) and binocular interactions (Baitch \& Levi, 1988, France \& Ver Hoeve, 1994), and has provided measures of the bandwidth and orientation selectivity of spatial-frequency channels in human vision (Regan \& Regan, 1987, 1988a). This latter application (in which superimposed spatial sinusoids were independently temporally modulated) is particularly noteworthy, since the bandwidths and orientation selectivities inferred from the VEP measures were in good agreement with the range of tuning of single neurons of macaque V1. This agreement not only validates the VEP approach, but also indicates that typical cortical neurons, though often considered quasilinear, are sufficiently nonlinear so as to generate intermodulation responses of high order.

As reported here, nearby bars sinusoidally modulated at distinct frequencies elicit VEPs with Fourier components at combination frequencies $n_{1} f_{1}+n_{2} f_{2}$, for $n_{1}$ and $n_{2}$ at least as high as 4 . The harmonics present in these interactions, and their dependence on temporal frequency and spatial configuration, provide information on the underlying neural interactions (Regan \& Regan, 1988a). To begin, we assume that signals from each component of the two-bar stimulus are initially processed linearly, resulting in signals that we denote as $Y_{1}(t)$ and $Y_{2}(t)$. If these signals $Y_{1}(t)$ and $Y_{2}(t)$ are then are combined in a product-like fashion, interaction terms will result. These resulting interaction terms are only of order 2 , that is, with $\left|n_{1}\right|=\left|n_{2}\right|=1$. Thus, a product of linearly filtered signals does not account for the third- and fourth-order interaction frequencies that we observe, and thus, a more complex model structure needs to be considered.

One possibility is that signals $Y_{1}(t)$ and $Y_{2}(t)$ from the two bars are indeed combined multiplicatively, but this combined signal is subject to a further nonlinearity. This will produce interaction terms of higher orders, but it cannot produce interactions at frequencies $n_{1} f_{1}+n_{2} f_{2}$ whose order $k=\left|n_{1}\right|+\left|n_{2}\right|$ is odd. (This can be seen by a simple symmetry argument. Under the above hypotheses, inverting the sign of both inputs inverts both $Y_{1}(t)$ and $Y_{2}(t)$ but leaves their product $Y_{1}(t) Y_{2}(t)$ unchanged. Since any nonlinear function of this product $N\left[Y_{1}(t) Y_{2}(t)\right]$ is also unchanged, it can only contain components that are even-order in the input. Thus, a late nonlinearity, coupled with a product-like interaction, cannot 

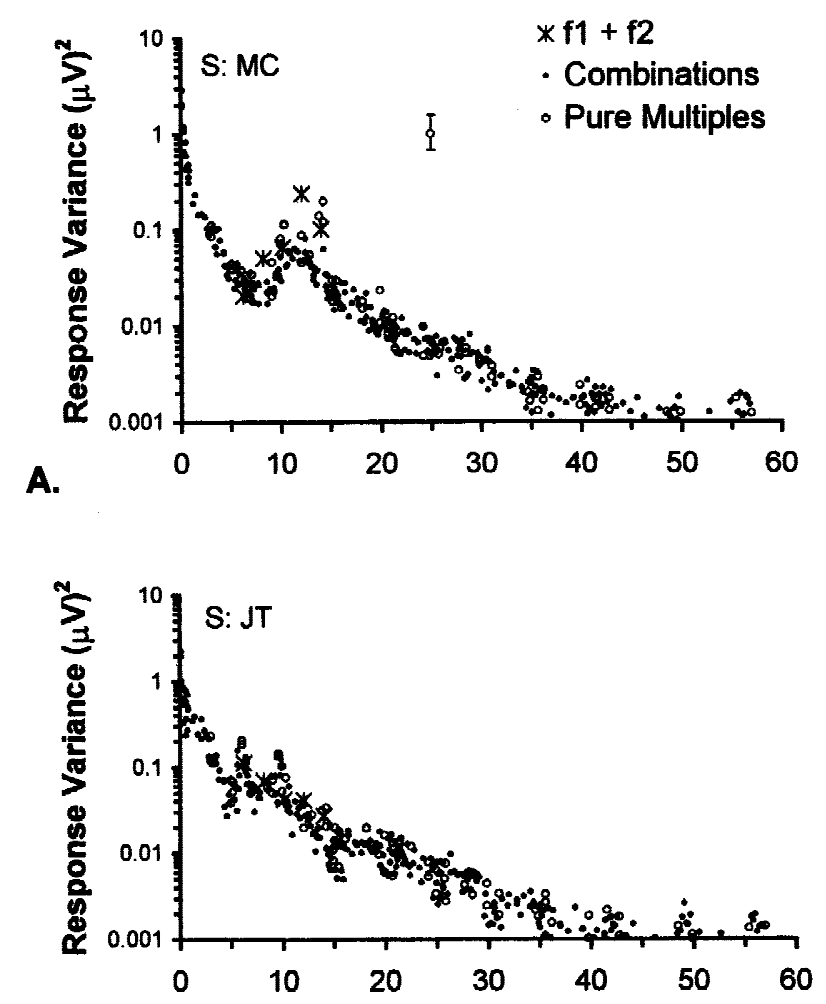

C.

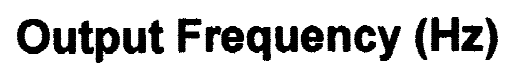

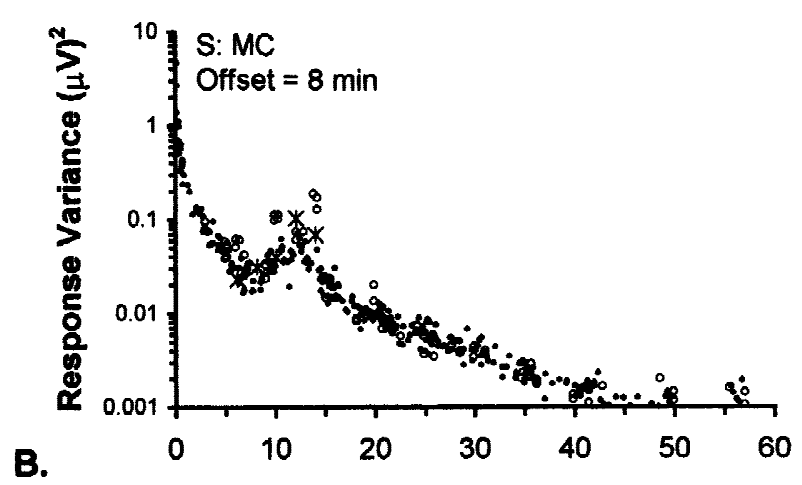

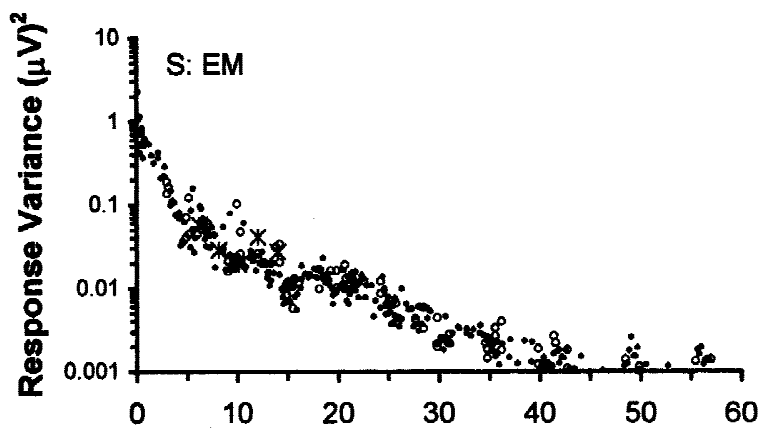

D.

Fig. 13. Undriven activity elicited by the Vernier target with and without Vernier displacement. Values up to order 8 of $P\left(n_{1}, n_{2}\right)$ are plotted as in Fig. 12. Each power spectrum includes data from five frequency pairs (those connected by a line in Fig. 2). Subjects MC (Panels A, B), JT (Panel C), and EM (Panel D). Vernier displacement $d=0$ in Panels A, C, and D; $d=8$ min in panel B.

account for the small but significant third-order responses we observed (Fig. 8).

Another possibility is that the interaction between the signals from the two bars can be described by linear filtering of signals from each bar to form the signals $Y_{1}(t)$ and $Y_{2}(t)$, followed by static nonlinear processing of the sum of these signals, that is, $N\left[Y_{1}(t)+Y_{2}(t)\right]$, followed by a final linear filtering $G$ of the combined signal. To determine whether this kind of structure can account for our results, we focus on the observation that the evenparity mixed fourth-order responses $A(2, \pm 2)$ are more prominent than the odd-parity mixed responses $A(3, \pm 1)$ and $A(1, \pm 3)$ (Fig. 9). For this analysis, we pool responses across displacements to increase signal-to-noise, since the systematic variation of the fourthorder responses with displacement is small.

In general, for the linear-nonlinear structure, the amplitude of a $k$ th-order interaction $A\left(n_{1}, n_{2}\right)$ depends on the output frequency $n_{1} f_{1}+n_{2} f_{2}$, the input frequencies $f_{1}$ and $f_{2}$, and on the integers $n_{1}$ and $n_{2}$ that combine to equal the order $k=\left|n_{1}\right|+\left|n_{2}\right|$ (Bedrosian \& Rice, 1971). Neglecting sixth and higher even-order responses, the amplitudes of the fourth-order responses have the approximate form

$$
\left|A\left(n_{1}, \pm n_{2}\right)\right| \approx K \frac{4 !}{n_{1} ! n_{2} !}\left|\tilde{Y}\left(f_{1}\right)\right|^{n_{1}}\left|\tilde{Y}\left(f_{2}\right)\right|^{n_{2}}\left|\widetilde{G}\left(n_{1} f_{1} \pm n_{2} f_{2}\right)\right|,
$$

where $\tilde{Y}\left(f_{1}\right)$ and $\tilde{Y}\left(f_{2}\right)$ represent the Fourier components at $f_{1}$ and $f_{2}$ that pass through the initial linear stage, $\widetilde{G}$ is the transfer func- tion of the final stage of linear filtering, and $K$ is a numerical factor that depends on the shape of the nonlinearity $N$. We do not have direct access to $K$ or to $\tilde{Y}$, but their roles can be eliminated by considering the index

$$
I=\log _{10}\left(\frac{4|A(2,2)|^{2}}{9|A(1,3)||A(3,1)|}\right) .
$$

The factor $4 / 9$ is introduced to compensate for the combinatorial factors in eqn. (4). The log is introduced to eliminate skewing in estimates of the index $I$, should any of the terms be near 0 . According to eqn. (4), the index $I$ for a linear-static nonlinear-linear model is approximated by

$$
I \approx \log _{10}\left(\frac{\left|\widetilde{G}\left(2 f_{1}+2 f_{2}\right)\right|^{2}}{\left|\widetilde{G}\left(f_{1}+3 f_{2}\right)\right|\left|\widetilde{G}\left(3 f_{1}+f_{2}\right)\right|}\right) .
$$

The role of $\widetilde{G}$ can be eliminated by focussing on the nearlymatched frequency pair $\left(f_{1}, f_{2}\right)=(3.027,3.223)$, since the relevant sum frequencies on the right side of eqn. (6) are nearly identical. (This would not have been the case for the analogous combination of difference frequencies, since $2 f_{1}-2 f_{2}$ is close to zero, but $f_{1}-$ $3 f_{2}$ and $3 f_{1}-f_{2}$ are not close to zero.) For closely spaced frequencies, the linear-nonlinear-linear model should yield an index $I$ whose expected value is 0 .

The drawback of this analysis is that the index $I$ is derived from a ratio of two quantities, each of which is not known very accurately, since many of the interaction components are small or not 
significantly different from zero. (Indeed, the small size of the terms in the denominator of eqn. (5) is what prompted this analysis.) It would be difficult to exclude datasets in which one or more of the component quantities was not "significant", since it is exactly this kind of behavior that is important for distinguishing the behavior of the linear-nonlinear-linear model from its alternatives. Fortunately, contamination of the measured responses by noise would tend to bias the index $I$ towards 0 , since noise would tend to contribute equally to the numerator and denominator of eqn. (5). Thus, examination of this index is a conservative test to examine the linear-nonlinear-linear hypothesis, but it is only useful if it shows a significant bias away from 0 . Moreover, a substantial scatter in values is to be expected.

Across the five subjects and two bar sizes, the ratio $I$ ranged from -0.60 to 1.00 (mean 0.27, S.E.M. 0.17). This is a large scatter, but nevertheless suggestive of a significant elevation above $0(P=0.07$ by $t$-test $)$, and thus a relative excess of even-even parity interactions above the expectation of a linear-nonlinearlinear model. The nature of the deviation, namely the prominence of even-parity mixed responses $A(2,2)$, suggests that even harmonics are generated before the signals from the two bars interact. If the frequencies $2 f_{1}$ and $2 f_{2}$ (but not $3 f_{1}$ and $3 f_{2}$ ) are already present in the signals $Y_{1}(t)$ and $Y_{2}(t)$ from the two bars, then multiplicative combination would generate even-parity mixed responses $A(2,2)$, but not the odd-parity mixed responses. Based on psychophysical studies, Victor and Conte (1999a) proposed a model to account for the dependence of Vernier threshold on stimulus dynamics, consisting of local processing followed by a productlike interaction across space. We showed that inclusion of partial rectification in the local processing stage was necessary to account for the dependence of Vernier threshold on relative phase and temporal frequency. The local rectification required by that model is consistent with the VEP evidence for local nonlinearities presented here.

The two-frequency stimulus did induce modest changes in the power spectrum (Fig. 12), thus indicating interactions of stimulusevoked responses with the background EEG. However, these changes were not specific to the "aligned" condition (Fig. 13). The changes consisted of alterations in background power at the stimulus frequencies and its harmonics, rather than the appearance of oscillations or high-frequency broadband activity. They were thus comparable to background interactions seen with sinusoidally reversing checkerboards (Mast \& Victor, 1991), and thus appear to be a general feature of EEG generation, rather than a change in endogenous activity associated with binding (Tallon-Baudry et al., 1996).

\section{Physiologic basis: Local interactions, not specific for} hyperacuity

The response properties of strongly oriented neurons in primary visual cortex likely play a critical role in Vernier acuity (Wilson, 1986; Carney et al., 1995; Swindale, 1995). As reported here, the Vernier displacements required to produce a significant change in the observed interactions was typically $4 \mathrm{~min}$ or more (Fig. 6), beyond the hyperacuity range and the psychophysical thresholds for these stimuli $(<0.5 \mathrm{~min}$, Victor \& Conte, 1999a). The size of this displacement corresponds to a rotation of the center-to-center line of the bars with respect to their horizontal axis by $4 \mathrm{deg}$. Orientation interactions that vary on this scale are not specific to Vernier tasks, but have also been observed for pairs of modulated gratings. For such stimuli, interaction responses fall by a factor of two with orientation shifts of only 6 deg (Regan \& Regan, 1987). This dependence is consistent with the tuning of cortical neurons that are selective for orientation (De Valois et al., 1982). Thus, one contributor to the observed interactions is the combination of signals between the two components of the stimulus within individual oriented receptive fields. The fact that we can only detect a change in the interaction response for displacements that are higher than Vernier thresholds is not in contradiction with the view that shortrange Vernier thresholds are determined by local orientation mechanisms, since our thresholds are limited by the ability to measure small VEP components within the noise of the ongoing EEG.

Several processes likely contribute to the interactions we observe. Colinear displacement of the bars to produce a gap (Fig. 10) alters the size of the interaction responses (Fig. 11 vs. Fig. 6). This dependence suggests contributions from longer-range interactions of like-oriented receptive fields, consistent with the notion of an "association field" that participates in contour integration (Field et al., 1993; Gilbert et al., 1996). Another kind of interaction that may contribute to our measurements is the short-range lateral interaction reported by Zemon and Ratliff (1982, 1984), which is sensitive to displacements under $1 \mathrm{~min}$. Because of the limitations of signal size, our VEP measurements cannot determine which of these components is responsible for the deviation from the linearnonlinear-linear structure.

\section{Comparison with other VEP correlates of Vernier acuity}

Previous workers have used other strategies to seek VEP components specific to Vernier acuity. Initial studies (Levi et al., 1983; Steinman et al., 1985; Zak \& Berkley, 1986) used a paradigm in which a short line segment abruptly broke colinearity with a longer stationary line. The resulting evoked potentials could be recorded for offsets as small as $10 \mathrm{~s}$ of arc. The VEP threshold correlated well with psychophysical thresholds, and showed degradation with interfering flankers (Steinman et al., 1985). Motion artifacts are a potential confound in such stimuli (Noss \& Srebro, 1996), but control conditions (Steinman et al., 1985) in which the moving segments were never in alignment ruled out the contribution of a simple movement artifact in these studies.

Norcia et al. (1999) and more recently Good and Norcia (2000) developed a steady-state version of this paradigm, based on colinearity break of strips of gratings. In the former studies, one stimulus component was static while the other oscillated in square-wave fashion; in the latter studies, motion of each stimulus component was driven by a different sinusoid. As in the earlier studies, thresholds for the interaction terms (obtained by extrapolation to zero amplitude from sweep-VEP data) correlated well with psychophysical thresholds. Response amplitudes were comparable to the amplitudes of the interactions reported here $(0.2$ to $0.5 \mu \mathrm{V})$.

The latency of the transient VEP response elicited by colinearity break is $200 \mathrm{~ms}$ or more (Levi et al., 1983; Steinman et al., 1985). (The phases or effective latencies of the steady-state colinearity break were not reported by Norcia et al., 1999.) This is much longer than the effective latencies of 145 to $165 \mathrm{~ms}$ that we found, both for the overall interactions and for the interactions that were displacement-dependent (Table 1). The difference in latencies suggests that the underlying mechanisms are somewhat distinct. In particular, the latencies we find are consistent with local processing in V1 and possibly V2, since latencies due to horizontal connections (Frégnac et al., 1996) would add about $30 \mathrm{~ms}$ to the P-100, as would intra-area delays (Bullier \& Novak, 1995). On the 
other hand, latencies of $200 \mathrm{~ms}$ or more suggest the contribution of cognitive stages, perhaps more closely linked to detecting a change in the stimulus than to hyperacuity per se (Regan, 1989).

Given the multiple mechanisms described above, the likely basis for the dissociation between our VEP findings and psychophysical thresholds is clear. In general, the spatial dependence of the interactions measured in the VEP likely reflect the characteristics of spatial pooling via cortical neurons. The slope of the pooling profile determines the extent to which the VEP signals have a measurable dependence on spatial interactions, but the height of this pooling profile might be more important for determining psychophysical thresholds. That is, the dissociation between our VEP indicators and behavior indicate that observers can parse specific components of spatial interactions, but passive summation of scalp signals cannot. Whether these other approaches have indeed extracted a VEP specific to Vernier acuity, or merely have an improved signal-to-noise, cannot as yet be determined: A comparison with the dependence on gap would help to indicate specificity of the VEP response, and measures of latency would help to assess the extent to which the VEP response is cognitive versus sensory.

\section{Acknowledgments}

We thank Jeremy Perlman for his assistance with VSG programming. A portion of this work was presented at the 1999 meeting of the Association for Research in Vision and Ophthalmology in Ft. Lauderdale, FL (Victor \& Conte, 1999b). This work was supported by NIH grant EY7977.

\section{References}

Baitch, L.W. \& Levi, D.M. (1988). Evidence for nonlinear binocular interactions in human visual cortex. Vision Research 28, 1139-1143.

BASAR, E. (1980). EEG-Brain Dynamics: Relation Between EEG and Brain Evoked Potentials. New York: Elsevier. 411 pp.

Bedrosian, E. \& Rice, S.O. (1971). The output properties of Volterra systems (nonlinear systems with memory) driven by harmonic and Gaussian inputs. Proceedings of the Institute of Electrical and Electronics Engineers 59, 1688-1707.

Bullier, J. \& NowaK, L.C. (1995). Parallel versus serial processing: New vistas on the distributed organization of the visual system. Current Opinion in Neurobiology 5, 497-503.

Carney, T., Silverstein, D.A. \& Klein, S.A. (1995). Vernier acuity during image rotation and translation: Visual performance limits. Vision Research 35, 1951-1964.

Carney, T. \& Klein, S.A. (1999). Optimal spatial localization is limited by contrast sensitivity. Vision Research 39, 503-511.

De Valois, R.L., Yund, E.W. \& Helper, N. (1982). The orientation and direction selectivity of cells in macaque visual cortex. Vision Research 22, 531-544

Field, D.J., Hayes, A. \& Hess, R.F. (1993). Contour integration by the human visual system: Evidence for a local "association field". Vision Research 33, 173-193.

France, T.D. \& Ver Hoeve, J.N. (1994). VECP evidence for binocular function in infantile esotropia. Journal of Pediatric Ophthalmology and Strabismus 31, 225-231.

Frégnac, Y., Bringuier, V., Chavane, F., Glaeser, L. \& Lorenceau, J. (1996). An intracellular study of space and time representation in primary visual cortical receptive fields. Journal of Physiology (Paris) 90, 189-197.

Gilbert, C.D., Das, A., Ito, M., Kapadia, M. \& Westheimer, G. (1996). Spatial integration and cortical dynamics. Proceedings of the National Academy of Sciences of the US.A. 93, 615-622.
Good, W.V. \& Norcia, A.M. (2000). A new Vernier acuity paradigm. Investigative Ophthalmology and Visual Sciences 41, S803.

KLEIN, S. A. \& LEVI, D.M. (1985). Hyperacuity thresholds of 1 sec: Theoretical predictions and empirical validation. Journal of the Optical Society of America A2, 1170-1190.

Krauskopf, J. \& Farell, B. (1991). Vernier acuity: Effects of chromatic content, blur, and contrast. Vision Research 31, 735-749.

Levi, D.M., Klein, S.A. \& CARneY, T. (2000). Unmasking the mechanisms for Vernier acuity: Evidence for a template model for Vernier acuity. Vision Research 40, 951-972.

Levi, D.M., Manny, R.E., Klein, S. \& Steinman, S.B. (1983). Electrophysiological correlates of hyperacuity in the human visual cortex. Nature 306, 468-470.

MAST, J. \& VICTOR, J.D. (1991). Fluctuations of steady-state VEP's: Interaction of driven evoked potentials and the EEG. Electroencephalography and Clinical Neurophysiology 78, 389-401.

Norcia, A.M., Wesemann, W. \& Manny, R.E. (1999). Electrophysiological correlates of Vernier and relative motion mechanisms in human visual cortex. Visual Neuroscience 16, 1123-1131.

Noss, R.S. \& Srebro, R. (1996). Apparent motion confounds early Vernier visual evoked potentials. Brain Research 727, 153-161.

Pfurtscheller, G. \& ANDrew, C. (1999). Event-related changes of band power and coherence: methodology and interpretation. Journal of Clinical Neurophysiology 16, 512-519.

Regan, D. (1989). Human Brain Electrophysiology. New York: Elsevier. $672 \mathrm{p}$.

Regan, D. \& Regan, M.P. (1987). Nonlinearity in human visual responses to two-dimensional patterns, and a limitation of Fourier methods. Vision Research 27, 2181-2183.

Regan, D. \& Regan, M.P. (1988a). Objective evidence for phaseindependent spatial frequency analysis in the human visual pathway. Vision Research 28, 187-191.

Regan, M.P. \& Regan, D. (1988b). A frequency domain technique for characterizing nonlinearities in biological systems. Journal of Theoretical Biology 133, 293-317.

REGAN, M.P. (1990). Theoretical analysis of the responses of rectifiers to a sum of two sinusoids input, and applications to modelling neural networks. Thesis, York University, North York, Ontario.

Shapley, R. \& Victor, J.D. (1986). Hyperacuity in cat retinal ganglion cells. Science 231, 999-1002.

Steinman, S.B., Levi, D.M., Klein, S.A. \& Manny, R. (1985). Selectivity of the evoked potential for Vernier offset. Vision Research $\mathbf{2 5}$, 951-962.

Swindale, N.V. (1995). Responses of neurons in cat striate cortex to Vernier offsets in reverse contrast stimuli. Visual Neuroscience 12, 805-817.

Tallon-Baudry, C., Bertrand, O., Delpuech, C. \& Pernier, J. (1996). Stimulus specificity of phase-locked and non-phase-locked $40 \mathrm{~Hz}$ visual responses in humans. Journal of Neuroscience 16, 4240-4249.

VICTOR, J.D. \& CONTE, M.M. (1999a). Short-range Vernier acuity: Interactions of temporal frequency, temporal phase, and stimulus polarity. Vision Research 39, 3351-3371.

Victor, J.D. \& Conte, M.M. (1999b). VEP analysis of the dynamics of Vernier acuity. (Abstract) Investigative Ophthalmology and Visual Sciences 40 (suppl.), 823.

VICTOR, J.D. \& MAST, J. (1991). A new statistic for steady-state evoked potentials. Electroencephalography and Clinical Neurophysiology 78, 378-388.

Westheimer, G. (1981). Visual hyperacuity. Progress in Sensory Physiology 1, 1-30.

WiLSON, H.R. (1986). Responses of spatial mechanisms can explain hyperacuity. Vision Research 26, 453-469.

ZAK, R. \& BERKLEY, M.A. (1986). Evoked potentials elicited by brief Vernier offsets: Estimating Vernier thresholds and properties of the neural substrate. Vision Research 26, 439-451.

Zemon, V. \& Ratliff, F. (1982). Visual evoked potentials: Evidence for lateral interactions. Proceedings of the National Academy of Sciences of the U.S.A. 79, 5723-5726.

Zemon, V. \& RATLifF, F. (1984). Intermodulation components of the visual evoked potential: Response to lateral and superimposed stimuli. Biological Cybernetics 50, 401-408. 